\title{
Quasicircles and bounded turning circles modulo bi-Lipschitz maps
}

\author{
David A. Herron and Daniel Meyer
}

This paper is dedicated to the fond memory of Fred Gehring, who helped so many of us come to love quasicircles.

\begin{abstract}
We construct a catalog, of snowflake type metric circles, that describes all metric quasicircles up to bi-Lipschitz equivalence. This is a metric space analog of a result due to Rohde. Our construction also works for all bounded turning metric circles; these need not be doubling. As a byproduct, we show that a metric quasicircle with Assouad dimension strictly less than two is bi-Lipschitz equivalent to a planar quasicircle.
\end{abstract}

\section{Introduction}

By definition, a metric quasicircle is the quasisymmetric image of the unit circle $\mathrm{S}^{1}$. (See Section 2 for definitions and basic terminology.) We exhibit a catalog that contains a bi-Lipschitz copy of each metric quasicircle. This is a metric space analog of recent work by Steffen Rohde [16], so we briefly describe his result. He constructed a collection $\mathcal{R}$ of snowflake type planar curves with the intriguing property that each planar quasicircle (the image of $S^{1}$ under a global quasiconformal selfhomeomorphism of the plane) is bi-Lipschitz equivalent to some curve in $\mathcal{R}$.

Rohde's catalog is $\mathcal{R}:=\bigcup \mathcal{R}_{p}$, where $p \in[1 / 4,1 / 2)$ is a snowflake parameter. Each curve in $\mathcal{R}_{p}$ is built in a manner reminiscent of the construction of the von Koch snowflake. Thus, each $R \in \mathcal{R}_{p}$ is the limit of a sequence $\left(R^{n}\right)$ of polygons where $R^{n+1}$ is obtained from $R^{n}$ by using the replacement rule illustrated in Figure 1: for each of the $4^{n}$ edges $E$ of $R^{n}$ we have two choices, either we replace $E$ with the four line segments obtained by dividing $E$ into four arcs of equal diameter, or we replace $E$ by a similarity copy of the polygonal arc $A_{p}$ pictured at the top right of Figure 1. In both cases $E$ is replaced by four new segments, each of these with diameter $(1 / 4) \operatorname{diam}(E)$ in the first case or with diameter $p \operatorname{diam}(E)$ in the second case. The second type of replacement is done so that the "tip" of

Mathematics Subject Classification (2010): Primary 30L10; Secondary 30C62,51F99.

Keywords: Quasicircle, Jordan curve, bounded turning, doubling. 


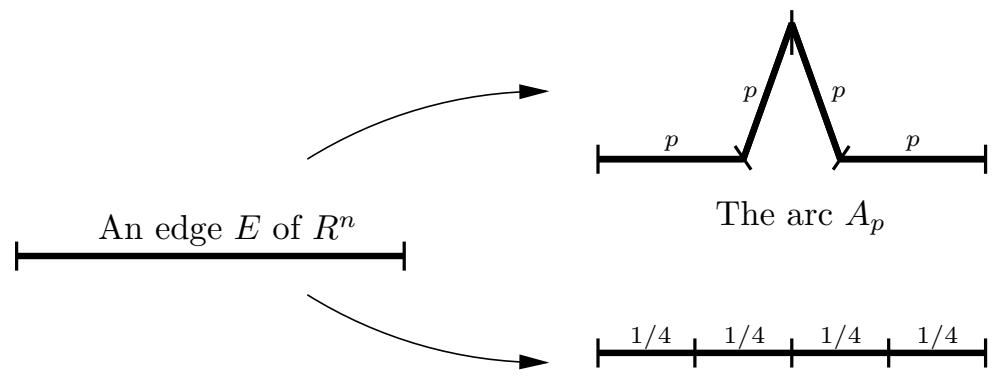

Figure 1. Construction of a Rohde-snowflake.

the replacement arc points into the exterior of $R^{n}$. This iterative process starts with $R^{1}$ being the unit square, and the snowflake parameter, thus the polygonal $\operatorname{arc} A_{p}$, is fixed throughout the construction. See the discussion at the beginning of $\S 4.3$ for more details.

The sequence $\left(R^{n}\right)$ of polygons converges, in the Hausdorff metric, to a planar quasicircle $R$ that we call a Rohde snowflake constructed with snowflake parameter $p$. Then $\mathcal{R}_{p}$ is the collection of all Rohde snowflakes that can be constructed with snowflake parameter $p$.

Rohde proved the following (see Theorem 1.1 in [16]):

A planar Jordan curve is a quasicircle if and only if it is the image of some Rohde snowflake under a bi-Lipschitz self-homeomorphism of the plane.

Thanks to a celebrated theorem of Ahlfors [1], there is a simple geometric criterion that characterizes planar quasicircles: a planar Jordan curve $\Gamma$ is a quasicircle if and only if it satisfies the bounded turning condition, which means that there is a constant $C \geq 1$ such that for each pair of points $x, y$ on $\Gamma$, the smaller diameter subarc $\Gamma[x, y]$ of $\Gamma$ that joins $x, y$ satisfies

$$
\operatorname{diam}(\Gamma[x, y]) \leq C|x-y|
$$

We say $\Gamma$ is $C$-bounded turning to emphasize the constant $C$.

Tukia and Väisälä [20] introduced the notion of a quasisymmetry between metric spaces. In this same paper they established the following metric space analog of Ahlfors' result:

A metric Jordan curve is a metric quasicircle if and only if it is both bounded turning and doubling (that is, of finite Assouad dimension).

Our catalog $\mathcal{S}$ of metric snowflake curves is a collection of metric circles $\left(\mathrm{S}^{1}, d\right)$ where the metrics $d$ are given in a simple way by specifying the diameter of each dyadic subarc of $\mathbf{S}^{1}$. See (3.1) and the end of $\S 3.2$ for precise details.

Our catalog is $\mathcal{S}:=\bigcup \mathcal{S}_{\sigma}$, and we also employ an auxiliary snowflake parameter $\sigma \in[1 / 2,1]$. Each $\left(\mathrm{S}^{1}, d_{\sigma}\right)$ in $\mathcal{S}_{\sigma}$ has a metric $d_{\sigma}$ that is obtained by the assignment of diameters to each dyadic subarc of $S^{1}$. As in Rohde's construction, at each step 
there are two choices: the diameter (with respect to $d_{\sigma}$ ) of a given dyadic subarc is either one-half, or $\sigma$, times the diameter of its parent subarc.

Each $\left(\mathrm{S}^{1}, d_{\sigma}\right)$ is a bounded turning circle. Moreover, when $\sigma<1,\left(\mathrm{~S}^{1}, d_{\sigma}\right)$ has Assouad dimension $\alpha \leq \log 2 / \log (1 / \sigma)<\infty$ (so, $2^{-1 / \alpha} \leq \sigma<1$ ), hence $\left(\mathrm{S}^{1}, d_{\sigma}\right)$ is doubling and thus a metric quasicircle; see Lemma 3.1(e). In fact, each collection $\mathcal{S}_{\sigma}$ (with $\sigma<1$ ) contains a bi-Lipschitz copy of every metric quasicircle with Assouad dimension strictly less than $\log (2) / \log (1 / \sigma)$. In addition, the subcatalog $\mathcal{S}_{1}$ contains a bi-Lipschitz copy of every bounded turning circle.

Here is our main result.

Theorem. Let $\Gamma$ be a metric Jordan curve.

(A) If $\Gamma$ is bounded turning, then $\Gamma$ is bi-Lipschitz equivalent to some curve in $\mathcal{S}_{1}$.

(B) If $\Gamma$ is a metric quasicircle with Assouad dimension $\alpha:=\operatorname{dim}_{\mathcal{A}}(\Gamma)$ and $\sigma \in\left(2^{-1 / \alpha}, 1\right)$, then $\Gamma$ is bi-Lipschitz equivalent to a curve in $\mathcal{S}_{\sigma}$.

(C) A metric quasicircle is bi-Lipschitz equivalent to a planar quasicircle if and only if it has Assouad dimension strictly less than two.

This result is quantitative in that the bi-Lipschitz constants depend only on the given data. For example, if $\Gamma$ is $C$-bounded turning, then the bi-Lipschitz constant in $(\mathrm{A})$ is

$$
L=8 C \max \left\{\operatorname{diam}(\Gamma), \operatorname{diam}(\Gamma)^{-1}\right\} .
$$

Minor modifications to our proofs reveal that the analogous results hold for bounded turning Jordan arcs and metric quasiarcs.

In addition, we explain how to recover Rohde's theorem from our result. This provides an alternative proof of Rohde's result that avoids the technical construction of a "uniform doubling measure" appearing as Theorem 1.2 in [16]. In view of this, our argument somewhat simplifies the proof of Rohde's theorem.

We mention that Bonk, Heinonen, and Rohde have established a result that gives metric quasicircles as metric boundaries of certain metric disks; see Lemma 3.7 in $[6]$.

The novel ideas in our approach include the following. We make extensive use of the fact that every bounded turning metric space is bi-Lipschitz equivalent to its associated diameter distance space; see Lemma 2.2. In particular, this permits us to restrict attention to 1-bounded turning Jordan curves. In this setting, the metrics are characterized, up to bi-Lipschitz equivalence, by knowledge of the diameters of certain subarcs, provided we have a sufficiently plentiful collection of subarcs; see Lemma 3.1. Finally, there is a straightforward way to build a bi-Lipschitz homeomorphism from one of our model curves onto such a metric Jordan curve; see Proposition 2.4 and Lemma 3.4.

This paper is organized as follows. Section 2 contains preliminary information including background material on Assouad dimension (in §2.2) and on quasisymmetric homeomorphisms (in $\S 2.3$ ). We prove a result about dividing an arc into subarcs of equal diameter (in $\S 2.5$ ) and (in $\S 2.6$ ) give a useful tool for constructing homeomorphisms between Jordan curves. We construct our dyadic models in $§ 3.2$ and prove our theorem in Section 4. 


\section{Preliminaries}

Here we set forth our (relatively standard) notation and terminology and present fundamental definitions and basic information. First we provide some background on quasisymmetric maps, doubling, and bounded turning. In $\S 2.4$ we show that we can restrict attention to 1-bounded turning circles. In $\S 2.5$ we prove that one can divide an arc into subarcs of equal diameter. In $\S 2.6$ we establish a useful proposition for constructing homeomorphisms between Jordan arcs or curves.

\subsection{Basic information}

For the record, $\mathbb{N}$ denotes the set of natural numbers, i.e., the positive integers.

We view the unit circle $S^{1}$ as the unit interval with its endpoints identified; that is, $\mathrm{S}^{1}=[0,1] /\{0 \sim 1\}=[0,1] / \sim$ where $s \sim t$ if and only if either $s=t$ or $\{s, t\}=\{0,1\}$. Then $\lambda$ denotes the (normalized) arc-length metric on $\mathrm{S}^{1}$ : for $s, t \in \mathrm{S}^{1}$ with say $0 \leq s \leq t \leq 1$,

$$
\lambda(s, t):=\min \{t-s, 1-(t-s)\} .
$$

A (closed) Jordan curve is the homeomorphic image of the circle $\mathrm{S}^{1}$ and a metric Jordan curve is a Jordan curve with a metric on it. A Jordan arc is the homeomorphic image of the unit interval $[0,1]$ and a metric Jordan arc is a Jordan arc with a metric on it. Thus Jordan curves and arcs are non-degenerate compact spaces, where non-degenerate means not a single point.

Given distinct points $x, y$ on a metric Jordan curve $\Gamma$, we write $\Gamma[x, y]$ to denote the closure of the smaller diameter component of $\Gamma \backslash\{x, y\}$; when both components have the same size, we randomly pick one. We often fix an orientation on $\Gamma$, and then $[x, y]$ stands for the subarc of $\Gamma$ that joins $x$ to $y$.

We note the following easy consequence of uniform continuity.

Lemma 2.1. Let $\Gamma$ be a metric Jordan curve or arc. Then for each $\varepsilon>0$, there are at most finitely many non-overlapping subarcs of $\Gamma$ that all have diameter at least $\varepsilon$.

Proof. Suppose $\Gamma=\varphi\left(\mathrm{S}^{1}\right)$ for some homeomorphism $\varphi$. Let $\varepsilon>0$ be given. Choose $\delta>0$ so that for each subarc $I \subset \mathrm{S}^{1}$ with $\operatorname{diam}_{\lambda}(I)<\delta$ we have $\operatorname{diam}(\varphi(I))<\varepsilon / 2$. Pick $N \in \mathbb{N}$ with $1 / N<\delta$. Partition $\mathrm{S}^{1}$ into adjacent equal length subarcs $I_{1}, \ldots, I_{N}$.

Let $A$ be a subarc of $\Gamma$ with $\operatorname{diam}(A) \geq \varepsilon$. Then $A$ must contain at least one of the subarcs $\varphi\left(I_{i}\right)$. Thus there are at most $N$ such subarcs $A$.

A similar argument applies when $\Gamma$ is an arc.

Throughout this article we employ the Polish notation $|x-y|$ for the distance between points $x, y$ in a metric space. The bounded turning condition (BT), also called Ahlfors' three point condition, makes sense in any connected metric space: this holds whenever points can be joined by continua whose diameters are no larger than a fixed constant times the distance between the original points. To be precise, given a constant $C \geq 1$, we say that $X$ has the $C$-bounded turning property if each 
pair of points $x, y \in X$ can be joined by a continuum $\Gamma[x, y]$ satisfying (BT). The bounded turning condition has a venerable position in quasiconformal analysis; see for example [20], [7], [14], [19] and the references therein.

A metric Jordan curve that is bounded turning is called a bounded turning circle, or a $C$-bounded turning circle if we wish to indicate the bounded turning constant $C$.

\subsection{Assouad dimension}

A metric space is doubling if there is a number $N$ such that every subset of diameter $D$ has a cover that consists of at most $N$ subsets each having diameter at most $D / 2$. It follows that every set of diameter $D$ has a cover by (at most) $N^{k}$ sets each of diameter at most $D / 2^{k}$.

The Assouad dimension $\operatorname{dim}_{\mathcal{A}}(X)$ of a metric space $X$ is the infimum of all numbers $\alpha>0$ with the property that there exists a constant $C>0$ such that for all $D>0$, each subset of diameter $D$ has a cover consisting of at most $C \varepsilon^{-\alpha}$ sets each of diameter at most $\varepsilon D$.

An equivalent description can be given in terms of separated sets. A subset $S \subset X$ is $r$-separated provided it is non-degenerate, meaning $\operatorname{card}(S)>1$, and for all distinct $x, y \in S,|x-y| \geq r$; in particular, $\operatorname{diam}(S) \geq r$. Then $\operatorname{dim}_{\mathcal{A}}(X)$ is the infimum of all numbers $\alpha>0$ with the property that there exists a constant $C>0$ such that for all $r>0$, each $r$-separated set $S \subset X$ has $\operatorname{card}(S) \leq C(\operatorname{diam}(S) / r)^{\alpha}$.

Evidently, a metric space has finite Assouad dimension if and only if it is doubling. The Assouad dimension was introduced by Assouad in [3] (see also [4]). A comprehensive overview is given in [11]. The role of doubling spaces in the general theory of quasisymmetric maps is explained in [10]. The Assouad dimension of a space is a bi-Lipschitz invariant, and it is always at least the Hausdorff dimension.

\subsection{Quasisymmetric homeomorphisms}

A homeomorphism $f: X \rightarrow Y$ of metric spaces $X, Y$ is called a quasisymmetry if there is a homeomorphism $\eta:[0, \infty) \rightarrow[0, \infty)$ such that for all distinct $x, y, z \in X$ and $t \in[0, \infty)$,

$$
\frac{|x-y|}{|x-z|} \leq t \quad \Longrightarrow \quad \frac{|f(x)-f(y)|}{|f(x)-f(z)|} \leq \eta(t) .
$$

This notion of quasisymmetry was introduced by Tukia and Väisälä in [20] where they also studied weak-quasisymmetries. A homeomorphism $f: X \rightarrow Y$ is a weak-quasisymmetry if there is a constant $H \geq 1$ such that, for all distinct $x, y, z \in X$,

$$
\frac{|x-y|}{|x-z|} \leq 1 \quad \Longrightarrow \quad \frac{|f(x)-f(y)|}{|f(x)-f(z)|} \leq H .
$$

Clearly every quasisymmetry is a weak-quasisymmetry. Tukia and Väisälä proved that each weak-quasisymmetry from a pseudo-convex space to a doubling space is a quasisymmetry; Heinonen has a similar result for maps from a connected doubling space to a doubling space; see Theorem 2.15 in [20] and Theorem 10.19 in [10]. In 
particular, this holds for maps between Euclidean spaces. However, a weakquasisymmetry may fail to be quasisymmetric if the target space is not doubling, as illustrated by an example in the paper by Tukia and Väisälä.

As discussed in the introduction, a metric quasicircle is the quasisymmetric image of $S^{1}$; thanks to work of Tukia and Väisälä, we know that these are precisely the doubling bounded turning circles. Recently, the second author [13] established the following characterization of bounded turning circles:

A metric Jordan curve is bounded turning if and only if it is a weakquasisymmetric image of the unit circle.

\subsection{Diameter distance}

Here we show that we can always restrict attention to 1-bounded turning circles. More precisely, we show that any bounded turning circle is bi-Lipschitz equivalent to a 1-bounded turning circle. The relevant tool employed is the notion of diameter distance $^{\dagger} \mathrm{dd}$, that is defined on any path connected metric space $(X,|\cdot|)$ by

$$
\operatorname{dd}(x, y):=\inf \{\operatorname{diam}(\gamma) \mid \gamma \text { a path in } X \text { joining } x, y\}
$$

It is not hard to see that $\mathrm{dd}$ is a metric on $X$. Here are some additional properties of dd.

Lemma 2.2. Let $(\Gamma,|\cdot|)$ be a metric Jordan curve or a metric Jordan arc and let $\mathrm{dd}$ be the associated diameter distance.

(a) The dd-diameter of any subarc $A$ of $\Gamma$ equals its diameter with respect to the original metric on $X$; that is, $\operatorname{diam}_{\mathrm{dd}}(A)=\operatorname{diam}(A)$.

(b) For all points $x, y \in \Gamma$, $\operatorname{diam}_{\mathrm{dd}}(\Gamma[x, y])=\mathrm{dd}(x, y)$. In particular, $(\Gamma, \mathrm{dd})$ is 1-bounded turning.

(c) $(\Gamma,|\cdot|)$ is $C$-bounded turning if and only if the identity $\operatorname{map}(\Gamma, \mathrm{dd}) \stackrel{\text { id }}{\rightarrow}(\Gamma,|\cdot|)$ is C-bi-Lipschitz.

Proof. To prove (a), first observe that for all $x, y \in \Gamma,|x-y| \leq \mathrm{dd}(x, y)$, so $\operatorname{diam}(A) \leq \operatorname{diam}_{\mathrm{dd}}(A)$. Next, for all $x, y \in A, \operatorname{dd}(x, y) \leq \operatorname{diam}(A)$, so $\operatorname{diam}_{\mathrm{dd}}(A) \leq$ $\operatorname{diam}(A)$.

Now (b) follows directly from (a) since

$$
\mathrm{dd}(x, y)=\operatorname{diam}(\Gamma[x, y])=\operatorname{diam}_{\mathrm{dd}}(\Gamma[x, y]) .
$$

It remains to establish $(\mathrm{c})$. If $(\Gamma,|\cdot|)$ is $C$-bounded turning, then for all $x, y \in \Gamma$

$$
\mathrm{dd}(x, y)=\operatorname{diam}(\Gamma[x, y]) \leq C|x-y| \leq C \mathrm{dd}(x, y),
$$

so the identity map is $C$-bi-Lipschitz.

Conversely, if this map is $C$-bi-Lipschitz, then for all $x, y \in \Gamma$

$$
\operatorname{diam}(\Gamma[x, y])=\operatorname{diam}_{\mathrm{dd}}(\Gamma[x, y])=\mathrm{dd}(x, y) \leq C|x-y|
$$

and therefore $(\Gamma,|\cdot|)$ is $C$-bounded turning.

\footnotetext{
${ }^{\dagger}$ This is also called inner diameter distance.
} 
We remark that in general the identity map $(X, \mathrm{dd}) \stackrel{\text { id }}{\rightarrow}(X,|\cdot|)$ need not be a homeomorphism. A simple example of this is the planar comb space

$$
X:=([0,1] \times\{0\}) \cup(\{0\} \times[0,1]) \bigcup_{n=1}^{\infty}(\{1 / n\} \times[0,1]) \subset \mathbb{R}^{2}
$$

equipped with Euclidean distance $|\cdot|$. If $z_{n}:=(1 / n, 1)$ and $a:=(0,1)$, then $\left|z_{n}-a\right| \rightarrow 0$ as $n \rightarrow \infty$, whereas $\mathrm{dd}\left(z_{n}, a\right) \geq 1$ for all $n$. Also, $(X,|\cdot|)$ is compact but $(X, \mathrm{dd})$ is not.

\subsection{Division of arcs}

Here we prove that any metric Jordan arc can be divided into any given number of subarcs each having exactly the same diameter.

The problem of finding points on a metric Jordan arc such that consecutive points are at the same distance is non-trivial. In 1930, Menger gave a proof that is short, simple and natural, but wrong; see page 487 in [12]. It was proved for arcs in Euclidean space in [2], and for the general case (indeed in more generality) as Theorem 3 in [17]; see also [22].

For the case at hand, i.e., for bounded turning arcs, it suffices to find adjacent subarcs that have equal diameter. We give the following elementary proof for this problem.

Proposition 2.3. Let $A$ be a metric Jordan arc and $N \geq 2$ an integer. Then we can divide $A$ into $N$ subarcs of equal diameter.

Proof. We may assume that $A$ is the unit interval $[0,1]$ equipped with some metric $d$. We claim that there are points $0=s_{0}<s_{1}<\cdots<s_{N-1}<s_{N}=1$ such that

$$
\operatorname{diam}\left[s_{0}, s_{1}\right]=\operatorname{diam}\left[s_{1}, s_{2}\right]=\cdots=\operatorname{diam}\left[s_{N-1}, s_{N}\right]
$$

where diam denotes diameter with respect to the metric $d$. When $N=2$ this follows by applying the Intermediate Value Theorem to the function $[0,1] \ni s \mapsto$ $\operatorname{diam}[0, s]-\operatorname{diam}[s, 1]$.

According to Lemma 2.2(a), we may replace $d$ by its associated diameter distance; thus we may assume from the start that for any $[s, t] \subset[0,1]$

$$
d(s, t)=\operatorname{diam}[s, t] .
$$

Next, we modify $d$ to get a metric $d_{\varepsilon}$ that is strictly increasing in the sense that

$$
[s, t] \subsetneq\left[s^{\prime}, t^{\prime}\right] \subset[0,1] \Longrightarrow d_{\varepsilon}(s, t)<d_{\varepsilon}\left(s^{\prime}, t^{\prime}\right)
$$

The crucial point here is the strict inequality, which need not hold in general.

To this end, fix $\varepsilon>0$ and for all $s, t \in[0,1]$ set

$$
d_{\varepsilon}(s, t):=d(s, t)+\varepsilon|t-s| .
$$


Then from (2.1) it follows that

$$
\operatorname{diam}_{\varepsilon}[s, t]=\operatorname{diam}[s, t]+\varepsilon|t-s|=d_{\varepsilon}(s, t),
$$

where $\operatorname{diam}_{\varepsilon}$ denotes diameter with respect to $d_{\varepsilon}$. This immediately implies (2.2).

We now show that $[0,1]$ can be divided into $N$ subintervals of equal $d_{\varepsilon}$-diameter. Consider the compact set $S:=\left\{\mathbf{s}=\left(s_{1}, \ldots, s_{N-1}\right) \mid 0 \leq s_{1} \leq \cdots \leq s_{N-1} \leq 1\right\}$. Set $s_{0}:=0, s_{N}:=1$. The function $\varphi: S \rightarrow \mathbb{R}$ defined by

$$
\varphi(\mathbf{s}):=\max _{0 \leq i \leq N-1} \operatorname{diam}_{\varepsilon}\left[s_{i}, s_{i+1}\right]-\min _{0 \leq j \leq N-1} \operatorname{diam}_{\varepsilon}\left[s_{j}, s_{j+1}\right]
$$

assumes a minimum on $S$. If this minimum is zero, we are done. Otherwise, there are adjacent intervals $\left[s_{i-1}, s_{i}\right],\left[s_{i}, s_{i+1}\right]$ that have different $d_{\varepsilon}$-diameter. Using the Intermediate Value Theorem as before, we can find $s_{i}^{\prime} \in\left[s_{i-1}, s_{i+1}\right]$ such that $\operatorname{diam}_{\varepsilon}\left[s_{i-1}, s_{i}^{\prime}\right]=\operatorname{diam}_{\varepsilon}\left[s_{i}^{\prime}, s_{i+1}\right]$. Then from (2.2) it follows that

$$
\begin{aligned}
\min _{0 \leq j<N} \operatorname{diam}_{\varepsilon}\left[s_{j}, s_{j+1}\right] & <\operatorname{diam}_{\varepsilon}\left[s_{i-1}, s_{i}^{\prime}\right] \\
& =\operatorname{diam}_{\varepsilon}\left[s_{i}^{\prime}, s_{i+1}\right]<\max _{0 \leq i<N} \operatorname{diam}_{\varepsilon}\left[s_{i}, s_{i+1}\right] .
\end{aligned}
$$

Applying this procedure to all subintervals of maximal $d_{\varepsilon}$-diameter we obtain a strictly smaller minimum for the function $\varphi$, which is impossible. Thus the minimum must be zero, and so we can subdivide [0,1] into $N$ subintervals of equal $d_{\varepsilon}$-diameter.

Consider now a sequence $\varepsilon_{n} \searrow 0$, as $n \rightarrow \infty$. Let $s_{1}^{n}<\cdots<s_{N-1}^{n}$ be the points that divide $[0,1]$ into $N$ subintervals of equal diameter with respect to $d_{\varepsilon_{n}}$. We can assume that for all $1 \leq j<N$, all points $s_{j}^{n}$ converge to $s_{j}$ as $n \rightarrow \infty$. It follows that for all $1 \leq i, j<N$,

$$
\operatorname{diam}\left[s_{i}, s_{i+1}\right]=\lim _{n \rightarrow \infty} \operatorname{diam}_{\varepsilon_{n}}\left[s_{i}^{n}, s_{i+1}^{n}\right]=\lim _{n \rightarrow \infty} \operatorname{diam}_{\varepsilon_{n}}\left[s_{j}^{n}, s_{j+1}^{n}\right]=\operatorname{diam}\left[s_{j}, s_{j+1}\right]
$$

as desired.

Proposition 2.3 is also true for metric Jordan curves $\Gamma$. In this case we are free to choose any point in $\Gamma$ to be an endpoint of one of the subarcs.

\subsection{Shrinking subdivisions}

Here we present a useful tool for constructing homeomorphisms between Jordan curves; see Proposition 2.4.

We begin with some terminology. Let $\Gamma$ be a metric Jordan curve or arc. A sequence $\left(\mathcal{A}^{n}\right)_{1}^{\infty}$ is a shrinking subdivision for $\Gamma$ provided:

- Each $\mathcal{A}^{n}$ is a finite decomposition of $\Gamma$ into compact arcs. Thus each $\mathcal{A}^{n}$ is a finite set of non-overlapping non-degenerate compact subarcs of $\Gamma$ that cover $\Gamma$. (Here non-overlapping means disjoint interiors and non-degenerate means not a single point.) 
- Each $\mathcal{A}^{n+1}$ is a subdivision of $\mathcal{A}^{n}$; i.e., for each arc $A$ in $\mathcal{A}^{n+1}$ there is a (unique) arc in $\mathcal{A}^{n}$, called the parent of $A$, that contains $A$.

- The subdivisions shrink, meaning that $\max _{A \in \mathcal{A}^{n}} \operatorname{diam}(A) \rightarrow 0$ as $n \rightarrow \infty$.

Assume $\left(\mathcal{A}^{n}\right)_{1}^{\infty}$ is a shrinking subdivision for $\Gamma$. We call $\left(A^{n}\right)_{1}^{\infty}$ a descendant sequence if $A^{1} \supset A^{2} \supset \cdots$, and $A^{n} \in \mathcal{A}^{n}$ for all $n \in \mathbb{N}$; thus each $A^{n}$ is the parent of $A^{n+1}$. Note that for any descendant sequence $\left(A^{n}\right)_{1}^{\infty}, \bigcap_{1}^{\infty} A^{n}$ is a single point. Also, for each point $x \in \Gamma$, there exists a descendant sequence $\left(A_{x}^{n}\right)_{1}^{\infty}$ with $\{x\}=\bigcap_{1}^{\infty} A_{x}^{n}$; such a descendant sequence need not be unique, but there can be at most two such sequences.

Shrinking subdivisions are useful for constructing homeomorphisms between metric Jordan curves; see $\S 4.1, \S 4.2$ and $\S 4.3$.

Proposition 2.4. Let $\mathrm{A}$ and $\mathrm{B}$ both be metric Jordan curves or metric Jordan arcs. Suppose $\left(\mathcal{A}^{n}\right)_{1}^{\infty}$ and $\left(\mathcal{B}^{n}\right)_{1}^{\infty}$ are shrinking subdivisions for $\mathrm{A}$ and $\mathrm{B}$ respectively. Assume these subdivisions are combinatorially equivalent, meaning that for each $n \in \mathbb{N}$ there are bijective maps $\Phi^{n}: \mathcal{A}^{n} \rightarrow \mathcal{B}^{n}$ such that for all $A, \tilde{A} \in \mathcal{A}^{n}$ and $A_{0} \in \mathcal{A}^{n+1}$,

$$
\begin{aligned}
A \cap \tilde{A}=\emptyset & \Longleftrightarrow \quad \Phi^{n}(A) \cap \Phi^{n}(\tilde{A})=\emptyset, \\
A_{0} \subset A & \Longleftrightarrow \quad \Phi^{n+1}\left(A_{0}\right) \subset \Phi^{n}(A) .
\end{aligned}
$$

Then the sequence $\left(\Phi^{n}\right)_{1}^{\infty}$ induces a homeomorphism $\mathrm{A} \stackrel{\varphi}{\rightarrow} \mathrm{B}$ with the property that

$$
\text { for all } n \in \mathbb{N} \text { and all } A \in \mathcal{A}^{n}, \quad \varphi(A)=\Phi^{n}(A) .
$$

Proof. Let $a \in \mathrm{A}$ and select a descendant sequence $\left(A^{n}\right)_{1}^{\infty}$ with $\{a\}=\bigcap_{1}^{\infty} A^{n}$. Setting $B^{n}:=\Phi^{n}\left(A^{n}\right)$ we obtain a descendant sequence $\left(B^{n}\right)_{1}^{\infty}$ with, say, $\{b\}:=$ $\bigcap_{1}^{\infty} B^{n}$. Suppose $\left(\tilde{A}^{n}\right)_{1}^{\infty}$ is a second descendant sequence with $\{a\}=\bigcap_{1}^{\infty} \tilde{A}^{n}$. Let $\tilde{B}^{n}:=\Phi^{n}\left(\tilde{A}^{n}\right)$ and $\{\tilde{b}\}=\bigcap_{1}^{\infty} \tilde{B}^{n}$. Since $A^{n} \cap \tilde{A}^{n} \neq \emptyset, B^{n} \cap \tilde{B}^{n} \neq \emptyset$ and therefore

$$
|b-\tilde{b}| \leq \operatorname{diam}\left(B^{n}\right)+\operatorname{diam}\left(\tilde{B}^{n}\right) \rightarrow 0 \quad \text { as } n \rightarrow \infty .
$$

Thus $\tilde{b}=b$ and so there is a well defined map $\varphi: \mathrm{A} \rightarrow \mathrm{B}$ given by setting $\varphi(a):=b$.

Two distinct points $a_{1}, a_{2} \in \mathrm{A}$ lie in disjoint arcs $A_{1}, A_{2} \in \mathcal{A}^{n}$, for sufficiently large $n \in \mathbb{N}$, and then $\varphi\left(A_{1}\right) \cap \varphi\left(A_{2}\right)=\emptyset$, so $\varphi\left(a_{1}\right) \neq \varphi\left(a_{2}\right)$ verifying that $\varphi$ is injective.

Given $b \in \mathrm{B}$ and a descendant sequence $\left(B^{n}\right)_{1}^{\infty}$ with $\{b\}=\bigcap_{1}^{\infty} B^{n}, A^{n}:=$ $\left(\Phi^{n}\right)^{-1}\left(B^{n}\right)$ defines a descendant sequence $\left(A^{n}\right)_{1}^{\infty}$ with, say, $\{a\}:=\bigcap_{1}^{\infty} A^{n}$, and then $\varphi(a)=b$. Thus $\varphi$ is surjective.

Let $\varepsilon>0$ be arbitrary. Fix an $n \in \mathbb{N}$ such that $\max \left\{\operatorname{diam}(B) \mid B \in \mathcal{B}^{n}\right\}<\varepsilon / 2$. Let $\delta:=\min \left\{\operatorname{dist}\left(A_{1}, A_{2}\right) \mid A_{1}, A_{2} \in \mathcal{A}^{n} ; A_{1} \cap A_{2}=\emptyset\right\}$. Suppose $a_{1}, a_{2} \in \mathcal{A}$ with $\left|a_{1}-a_{2}\right|<\delta$. Pick $A_{k} \in \mathcal{A}^{n}$ with $a_{k} \in \mathrm{A}_{k}$. The definition of $\delta$ ensures that $A_{1} \cap A_{2} \neq \emptyset$. Therefore,

$$
\left|\varphi\left(a_{1}\right)-\varphi\left(a_{2}\right)\right| \leq \operatorname{diam}\left(\varphi\left(A_{1}\right)\right)+\operatorname{diam}\left(\varphi\left(A_{2}\right)\right) \leq \varepsilon
$$

and so $\varphi$ is (uniformly) continuous and hence a homeomorphism. 


\section{Dyadic subarcs and diameter functions}

Here we give precise definitions of our model curves, i.e., our model circles. These are given by defining metrics on $S^{1}$. Since we can restrict attention to 1-bounded turning circles (thanks to Lemma 2.2(b,c)), it suffices to only know the diameters of certain subarcs, provided we have a sufficiently plentiful collection of subarcs; for this purpose we use the dyadic subarcs described in $\S 3.1$. We introduce the notion of a dyadic diameter function in $\S 3.2$; these provide a simple method for constructing metrics on $\mathrm{S}^{1}$. Then in $\S 3.4$ we establish a convenient way to detect when two such metrics are bi-Lipschitz equivalent, and also when a given metric Jordan curve is bi-Lipschitz equivalent to $S^{1}$ with such a metric.

\subsection{Dyadic subarcs}

With our convention that $\mathrm{S}^{1}=[0,1] /\{0 \sim 1\}$, the $n^{\text {th }}$-generation dyadic subarcs of $S^{1}$ (obtained by dividing $S^{1}$ into $2^{n}$ subarcs of equal diameter) are the subarcs of the form

$$
I_{k}^{n}:=\left[k / 2^{n},(k+1) / 2^{n}\right], \quad \text { where } k \in\left\{0,1, \ldots, 2^{n}-1\right\} .
$$

Noting that $I^{0}:=I_{0}^{0}:=\mathrm{S}^{1}$, we define

$$
\mathcal{I}^{n}:=\left\{I_{k}^{n} \mid k \in\left\{0,1, \ldots, 2^{n}-1\right\}\right\} \quad \text { and then } \mathcal{I}:=\bigcup_{n=0}^{\infty} \mathcal{I}^{n} .
$$

Each dyadic subarc $I^{n} \in \mathcal{I}^{n}$ contains exactly two $I^{n+1}, \tilde{I}^{n+1} \in \mathcal{I}^{n+1}$ that we call the children of $I^{n}$, and then $I^{n}$ is the parent of each of $I^{n+1}, \tilde{I}^{n+1}$.

It is convenient to introduce some terminology. Often, we denote the children or sibling or parent of a generic $I \in \mathcal{I}$ by

$$
I_{0}, I_{1} \text { or } \tilde{I} \text { or } \hat{I}
$$

respectively; implicit in the use of the latter two notations is the requirement that $I \neq \mathrm{S}^{1}$.

Clearly, $\left(\mathcal{I}^{n}\right)_{1}^{\infty}$ is a shrinking subdivision for $\mathrm{S}^{1}$ in the sense of $\S 2.6$. Recall too that a sequence $\left(I^{n}\right)_{n=0}^{\infty}$ of dyadic subarcs $I^{n} \in \mathcal{I}^{n}$ is a descendant sequence provided $I^{0} \supset I^{1} \supset I^{2} \supset \cdots$; that is, for each $n, I^{n+1}$ is a child of $I^{n}$. We note that for each $x \in \mathrm{S}^{1}$ there is a descendant sequence $\left(I_{x}^{n}\right)_{n=0}^{\infty}$ with $\{x\}=\bigcap_{n=0}^{\infty} I_{x}^{n}$; such a sequence is unique unless $x$ is a dyadic endpoint in which case there are exactly two such sequences.

By connecting each arc to its parent, we can view $\mathcal{I}$ as the vertex set of a rooted binary tree. In this connection, we use the following elementary fact on various subtrees:

König's Lemma. A rooted tree with infinitely many vertices, each of finite degree, contains an infinite simple path.

In our setting this means that each infinite subtree contains a descendant sequence. 
In the proof of part (B) of our Theorem it will be convenient to "do $m$ steps at once". This means that instead of dividing an arc into two subarcs, we will divide it into $2^{m}$ subarcs. With this in mind, we also consider the family $\mathcal{J}$ of all $2^{m}$-adic subarcs; thus

$$
\mathcal{J}:=\bigcup_{n=0}^{\infty} \mathcal{J}^{n}, \quad \text { where } \mathcal{J}^{n}=\mathcal{I}^{m n} .
$$

Each $\mathcal{J}^{n}$ contains the $2^{m n}$ subarcs of the form $J_{k}^{n}:=\left[k / 2^{m n},(k+1) / 2^{m n}\right]$ in $\mathcal{I}^{m n}$ with $k \in\left\{0,1, \ldots, 2^{m n}-1\right\}$. Each such arc $J^{n}$ has $2^{m}$ children, i.e., arcs $J^{n+1} \in \mathcal{I}^{m(n+1)}$, all of which are contained in $J^{n}$.

\subsection{Dyadic diameter functions}

A dyadic diameter function $\Delta$ assigns a diameter $\Delta(I)$ to each dyadic subarc $I \in \mathcal{I}$. More precisely, we call $\Delta: \mathcal{I} \rightarrow(0,1]$ a dyadic diameter function constructed using the snowflake parameter $\sigma \in[1 / 2,1]$ provided $\Delta\left(\mathrm{S}^{1}\right)=1$ and

$$
\forall I \in \mathcal{I}, \quad \text { either } \quad \Delta\left(I_{0}\right)=\Delta\left(I_{1}\right):=\frac{1}{2} \Delta(I) \quad \text { or } \quad \Delta\left(I_{0}\right)=\Delta\left(I_{1}\right):=\sigma \Delta(I),
$$

where $I_{0}, I_{1}$ are the two children of $I$. When $\sigma=1$, we also require

$$
\lim _{n \rightarrow \infty} \max \left\{\Delta(I) \mid I \in \mathcal{I}^{n}\right\}=0 .
$$

If $\sigma<1$, this latter condition is automatically true. The snowflake parameter $\sigma$ is kept fixed throughout the construction.

Each dyadic diameter function $\Delta$ produces a distance function $d=d_{\Delta}$ on $\mathrm{S}^{1}$ defined by

$$
d(x, y)=d_{\Delta}(x, y):=\inf \sum_{k=1}^{N} \Delta\left(I_{k}\right)
$$

where the infimum is taken over all $x y$-chains $I_{1}, \ldots, I_{N}$ in $\mathcal{I}$; thus $x$ and $y$ lie in $I_{1} \cup \cdots \cup I_{N}$, each $I_{k}$ belongs to $\mathcal{I}$, and for all $2 \leq k \leq N, I_{k-1} \cap I_{k} \neq \emptyset$.

Now we present various properties of this metric. Our 'diameter function' terminology is motivated by item $(\mathrm{d})$ below.

Lemma 3.1. Let $\mathcal{I} \stackrel{\Delta}{\rightarrow}(0, \infty)$ be a dyadic diameter function and define $d:=d_{\Delta}$ as in (3.1). Then:

(a) $d$ is a metric on $\mathrm{S}^{1}$.

(b) The identity map id : $\left(\mathrm{S}^{1}, d\right) \rightarrow\left(\mathrm{S}^{1}, \lambda\right)$ is a 1-Lipschitz homeomorphism; recall that $\lambda$ is the normalized length metric on $\mathrm{S}^{1}$; see $\S 2.1$.

(c) $\left(\mathrm{S}^{1}, d\right)$ is 1-bounded turning (so d is its own diameter distance).

(d) The diameter (with respect to $d$ ) of each dyadic subarc is given by $\Delta$; i.e., for all $n \in \mathbb{N}$ and all $I \in \mathcal{I}^{n}$, $\operatorname{diam}_{d}(I)=\Delta(I)$. 
(e) If $\Delta$ is constructed using a snowflake parameter $\sigma \in[1 / 2,1)$, then the Assouad dimension of $\left(\mathrm{S}^{1}, d\right)$ is at most $\log 2 / \log (1 / \sigma)$. Equality holds for the "extremal model" where we take $\Delta\left(I_{0}\right)=\Delta\left(I_{1}\right)=\sigma \Delta(I)$ for both children $I_{0}, I_{1}$ of each $I \in \mathcal{I}$.

Proof. (a) It is clear that $d$ is non-negative, symmetric, and satisfies the triangle inequality. Given $x \in \mathrm{S}^{1}$ and $n \in \mathbb{N}$, let $I_{x}^{n} \in \mathcal{I}^{n}$ be a dyadic subarc containing $x$. Since $\left(I_{x}^{n}\right)_{1}^{\infty}$ is an $x x$-chain, $d(x, x) \leq \Delta\left(I_{x}^{n}\right) \rightarrow 0$ (as $\left.n \rightarrow \infty\right)$, so $d(x, x)=0$. Since $\Delta\left(I^{n}\right) \geq 2^{-n}=\operatorname{diam}_{\lambda}\left(I^{n}\right)$, it follows that $d(x, y) \geq \lambda(x, y)$. Thus $d(x, y)=0$ if and only if $x=y$.

(b) This follows from Proposition 2.4 and the penultimate sentence in the proof of $(\mathrm{a})$.

(d) Fix $I \in \mathcal{I}^{n}$ with $n \geq 1$. For all points $x, y \in I, I$ is an $x y$-chain, so $d(x, y) \leq \Delta(I)$ and thus $\operatorname{diam}_{d}(I) \leq \Delta(I)$. The opposite inequality follows from the observation that any chain joining the endpoints of $I$ must cover either $I$ or its sibling $\tilde{I}$.

(c) To prove that $\left(\mathrm{S}^{1}, d\right)$ is 1-bounded turning, fix distinct points $x, y \in \mathrm{S}^{1}$. Let $[x, y]$ and $[y, x]$ be the two closed arcs on $\mathrm{S}^{1}$ between $x, y$ (i.e., the closures of the components of $\left.\mathrm{S}^{1} \backslash\{x, y\}\right)$. Assume that $\operatorname{diam}_{d}([x, y]) \leq \operatorname{diam}_{d}([y, x])$. Next let $I_{1}, \ldots, I_{N}$ be any $x y$-chain. Then $I_{1} \cup \cdots \cup I_{N} \supset A$, where either $A=[x, y]$ or $A=[y, x]$, so $\operatorname{diam}_{d}([x, y]) \leq \operatorname{diam}_{d}(A)$.

For any $a, b \in A, I_{1}, \ldots, I_{N}$ is an $a b$-chain; therefore

$$
d(a, b) \leq \sum_{n=1}^{N} \Delta\left(I_{n}\right), \text { and thus } \operatorname{diam}_{d}([x, y]) \leq \operatorname{diam}_{d}(A) \leq \sum_{n=1}^{N} \Delta\left(I_{n}\right) .
$$

Taking the infimum over all such $x y$-chains $I_{1}, \ldots, I_{N}$ yields

$$
\operatorname{diam}_{d}([x, y]) \leq d(x, y) .
$$

(e) First, suppose $\Delta$ is constructed using a snowflake parameter $\sigma \in[1 / 2,1)$. Let $\alpha:=\log 2 / \log (1 / \sigma)$, so $\sigma^{-\alpha}=2$. Fix an arbitrary $\varepsilon \in(0,1]$. Choose $n \in \mathbb{N}$ so that $\sigma^{n}<\varepsilon \leq \sigma^{n-1}$. Consider a dyadic subarc $I^{n} \in \mathcal{I}^{n}$. Then $\operatorname{diam}_{d}\left(I^{n}\right)=$ $\Delta\left(I^{n}\right) \leq \sigma^{n}<\varepsilon$.

Now let $A$ be any $\varepsilon$-separated set in $\left(\mathrm{S}^{1}, d\right)$. Then $A$ contains at most one point in each dyadic subarc $I^{n} \in \mathcal{I}^{n}$. Thus

$$
\operatorname{card}(A) \leq 2^{n}=\left(\sigma^{-\alpha}\right)^{n}=\sigma^{-\alpha}\left(\sigma^{n-1}\right)^{-\alpha} \leq 2 \varepsilon^{-\alpha} .
$$

It follows that the Assouad dimension of $\left(\mathrm{S}^{1}, d\right)$ is at most $\alpha$; see $\S 2.2$.

Finally, consider the dyadic diameter function given by setting $\Delta\left(I^{n+1}\right):=$ $\sigma \Delta\left(I^{n}\right)$ (for each child $I^{n+1} \in \mathcal{I}^{n+1}$ of every $I^{n} \in \mathcal{I}^{n}$ ) and its corresponding metric $d=d_{\Delta}$. Then for each $n \in \mathbb{N}$, the set $A^{n}:=\left\{k / 2^{n} \mid 0 \leq k<2^{n}\right\}$ of $n^{\text {th }}$-generation endpoints is $\sigma^{n}$-separated in $\left(\mathrm{S}^{1}, d\right)$. Assume constants $C>0, \alpha>0$ are given so that the number of $\varepsilon$-separated points is at most $C \varepsilon^{-\alpha}$. Taking $\varepsilon:=\sigma^{n}$ we obtain

$$
C \varepsilon^{-\alpha}=C\left(\sigma^{n}\right)^{-\alpha}=C\left(\sigma^{-\alpha}\right)^{n} \geq \operatorname{card}\left(A^{n}\right)=2^{n}, \text { so } \alpha \geq \frac{\log 2}{\log (1 / \sigma)} .
$$


Given $\sigma \in[1 / 2,1]$, we let $\mathcal{S}_{\sigma}$ be the collection of all metric circles $\left(\mathrm{S}^{1}, d\right)$, where the metric $d=d_{\Delta}$ is defined as in $(3.1)$ and $\Delta: \mathcal{I} \rightarrow(0,1]$ is any dyadic diameter function constructed using the snowflake parameter $\sigma$. Then

$$
\mathcal{S}:=\bigcup_{\sigma \in[1 / 2,1]} \mathcal{S}_{\sigma}
$$

is our catalog of snowflake type metric circles. Thanks to the Tukia-Väisälä characterization, Lemma 3.1(c,e) imply that for $\sigma \in[1 / 2,1)$, each curve in $\mathcal{S}_{\sigma}$ is a metric quasicircle.

The curves in $\mathcal{S}_{1}$ are bounded turning circles, but need not be metric quasicircles since they may fail to be doubling. There is a simple test for doubling that we give below in Lemma 3.7.

\section{3. $2^{m}$-adic diameter functions}

We also require $2^{m}$-adic diameter functions; recall (see the end of $\S 3.1$ ) that $\mathcal{J}$ denotes the family of $2^{m}$-adic subarcs of $\mathrm{S}^{1}$. We call $\Delta: \mathcal{J} \rightarrow(0,1]$ a $2^{m}$-adic diameter function constructed using the snowflake parameter $\tau \in\left[1 / 2^{m}, 1\right]$ provided $\Delta\left(\mathrm{S}^{1}\right)=1$ and

$$
\begin{aligned}
\forall J \in \mathcal{J}, \quad \text { either } \quad \Delta\left(J_{0}\right)=\Delta\left(J_{1}\right)=\cdots=\Delta\left(J_{2^{m}-1}\right):=\frac{1}{2^{m}} \Delta(J) \\
\text { or } \quad \Delta\left(J_{0}\right)=\Delta\left(J_{1}\right)=\cdots=\Delta\left(J_{2^{m}-1}\right):=\tau \Delta(J),
\end{aligned}
$$

where $J_{0}, \ldots, J_{2^{m}-1}$ are the children of $J$. The snowflake parameter $\tau$ is fixed throughout the construction. If $\tau=1$, we also require

$$
\lim _{n \rightarrow \infty} \max \left\{\Delta(J) \mid J \in \mathcal{J}^{n}\right\}=0 .
$$

When $\tau<1$ this latter condition is automatically true.

Just as for dyadic diameter functions, each $2^{m}$-adic diameter function $\Delta$ has an associated distance function $d_{\Delta}$ defined as in (3.1) but now we only consider $x y$ chains chosen from $\mathcal{J}$. Lemma 3.1 remains valid for $2^{m}$-adic diameter functions; however, in part (e) we must take $\sigma=\tau^{1 / m}$, where the $2^{m}$-adic diameter function is constructed using the snowflake parameter $\tau \in\left[1 / 2^{m}, 1\right]$.

We note the following useful fact. For each dyadic arc $I \in \mathcal{I}$, there exist $2^{m}$-adic $\operatorname{arcs} J^{n} \in \mathcal{J}^{n}$ and $J^{n+1} \in \mathcal{J}^{n+1}$ such that

$$
J^{n+1} \subset I \subset J^{n} .
$$

Each $2^{m}$-adic diameter function $\Delta: \mathcal{J} \rightarrow(0,1]$, with snowflake parameter $\tau$, has a natural extension to a dyadic diameter function $\Delta: \mathcal{I} \rightarrow(0,1]$, with snowflake parameter $\sigma:=\tau^{1 / m}$, that is defined as follows. Fix a subarc $J^{n} \in \mathcal{J}$ and let $J^{n+1} \subset J^{n}$ be any child of $J^{n}$. Let $J^{n}=: I^{m n} \supset I^{m n+1} \supset \cdots \supset I^{m(n+1)}:=J^{n+1}$ be the finite descendant sequence from $\mathcal{I}$ determined by $J^{n+1}$ and $J^{n}$. Set

$$
\rho:=\left[\Delta\left(J^{n+1}\right) / \Delta\left(J^{n}\right)\right]^{1 / m} \quad\left(\text { so, } \rho \in\left\{1 / 2, \tau^{1 / m}\right\}\right)
$$


and for each $i \in\{0,1, \ldots, m\}$ define

$$
\Delta\left(I^{m n+i}\right):=\rho^{i} \Delta\left(J^{n}\right)
$$

In view of (3.2), this procedure defines $\Delta(I)$ for each $I \in \mathcal{I}$. Note that $\Delta\left(I^{m n+0}\right)=$ $\Delta\left(J^{n}\right)$ and $\Delta\left(I^{m n+m}\right)=\Delta\left(J^{n+1}\right)$, so $\Delta: \mathcal{I} \rightarrow(0,1]$ is an extension of $\Delta: \mathcal{J} \rightarrow$ $(0,1]$. Clearly this extension is a dyadic diameter function constructed with the snowflake parameter $\sigma=\tau^{1 / m}$.

Lemma 3.2. Let $\Delta: \mathcal{J} \rightarrow(0,1]$ be a $2^{m}$-dyadic diameter function that has been extended to all dyadic intervals, i.e., to a dyadic diameter function $\Delta: \mathcal{I} \rightarrow(0,1]$, as described above. Let $d_{\mathcal{I}}$ and $d_{\mathcal{J}}$ be the metrics defined via $\left.\Delta\right|_{\mathcal{I}}$ and $\left.\Delta\right|_{\mathcal{J}}$ respectively, meaning by (3.1) and using chains from $\mathcal{I}$ and $\mathcal{J}$ respectively. Then, for all $x, y \in \mathrm{S}^{1}$,

$$
\frac{1}{2^{m}} d_{\mathcal{J}}(x, y) \leq d_{\mathcal{I}}(x, y) \leq d_{\mathcal{J}}(x, y)
$$

Proof. The righthand inequality holds because there are more $x y$-chains available when we use subarcs from $\mathcal{I}$. To prove the lefthand inequality, let $I_{1}, \ldots, I_{N}$ be an $x y$-chain from $\mathcal{I}$. Now use (3.2) to get a corresponding $x y$-chain $J_{1}, \ldots, J_{N}$ from $\mathcal{J}$ and with $J_{k}^{\prime} \subset I_{k} \subset J_{k}$ where $J_{k}^{\prime}$ is some child of $J_{k}$. Then for each $k$

$$
\Delta\left(I_{k}\right) \geq \Delta\left(J_{k}^{\prime}\right) \geq 2^{-m} \Delta\left(J_{k}\right), \quad \text { so } \quad d_{\mathcal{J}}(x, y) \leq \sum_{k=1}^{N} \Delta\left(J_{k}\right) \leq 2^{m} \sum_{k=1}^{N} \Delta\left(I_{k}\right)
$$

Taking an infimum gives $d_{\mathcal{J}}(x, y) \leq 2^{m} d_{\mathcal{I}}(x, y)$.

The previous lemma and prior discussion reveal that in order to prove that a given metric circle $(\Gamma,|\cdot|)$ is bi-Lipschitz equivalent to a curve in $\mathcal{S}_{\sigma}$, it is sufficient to construct a $2^{m}$-adic model circle (with snowflake parameter $\tau=\sigma^{m}$ ) that is biLipschitz equivalent to $(\Gamma,|\cdot|)$; this will yield a dyadic model circle (with snowflake parameter $\sigma$ ) bi-Lipschitz equivalent to $(\Gamma,|\cdot|)$.

Remark 3.3. Rohde's construction is based on 4-adic arcs rather than dyadic arcs. Results similar to the above also hold in this case. Namely each $4^{m}$-adic diameter function $\bigcup_{k} \mathcal{I}^{4^{m k}} \rightarrow(0,1]$, with snowflake parameter $\tau$ in $\left[1 / 4^{m}, 1\right]$, has an extension to a 4 -adic diameter function with snowflake parameter $\sigma:=\tau^{1 / m} \in$ $[1 / 4,1]$. The analog of Lemma 3.2 holds: the metrics constructed from these two diameter functions are bi-Lipschitz equivalent.

\subsection{Bi-Lipschitz equivalence}

Let $(\Gamma,|\cdot|)$ be a bounded turning circle and $\left(\mathrm{S}^{1}, d_{\Delta}\right)$ be a model circle where $\Delta$ is some dyadic diameter function. In the following we show that to prove bi-Lipschitz equivalence of $(\Gamma,|\cdot|)$ and $\left(\mathrm{S}^{1}, d_{\Delta}\right)$, it is enough to show bi-Lipschitz equivalence for dyadic subarcs. More precisely, we establish the following result: 
Lemma 3.4. Let $(\Gamma,|\cdot|)$ be a $C$-bounded turning circle and $d=d_{\Delta}$ a metric on $\mathrm{S}^{1}$ defined via a $2^{m}$-adic diameter function $\Delta$. Let $\varphi: S^{1} \rightarrow \Gamma$ be a homeomorphism. Suppose there exists a constant $K \geq 1$ such that, for all $J \in \mathcal{J}$,

$$
K^{-1} \operatorname{diam}(\varphi(J)) \leq \Delta(J) \leq K \operatorname{diam}(\varphi(J)) .
$$

Then $\left(\mathrm{S}^{1}, d\right) \stackrel{\varphi}{\rightarrow}(\Gamma,|\cdot|)$ is L-bi-Lipschitz, where $L:=2^{m+1} C K$.

Before proving this lemma (see Paragraph 3.6), we first give a simple way to estimate the diameter of an arc in terms of the diameters of dyadic subarcs.

Lemma 3.5. Let $\mathcal{J} \stackrel{\Delta}{\rightarrow}(0,1]$ be a $2^{m}$-adic diameter function with associated metric $d=d_{\Delta}$. For each arc $A \subset \mathrm{S}^{1}$, define

$$
\delta(A)=\delta_{\Delta}(A):=\max \{\Delta(I) \mid I \subset A, I \in \mathcal{J}\} .
$$

Then for all arcs $A \subset \mathrm{S}^{1}$,

$$
\delta(A) \leq \operatorname{diam}_{d}(A) \leq 2^{m+1} \delta(A) .
$$

In fact, there are $2^{m}$-adic arcs $I, J \in \mathcal{J}$ such that $I \cup J \subset A \subset \hat{I} \cup \hat{J}, \Delta(I)=\delta(A)$, and either $I=J$ or $\hat{I}, \hat{J}$ are adjacent. Here $\hat{I}, \hat{J} \in \mathcal{J}$ are the parents of $I, J$ relative to $\mathcal{J}$.

Proof. Let $A$ be a subarc of $\mathrm{S}^{1}$. Suppose we have verified the existence of the described $2^{m}$-adic $\operatorname{arcs} I, J \in \mathcal{J}$. Then

$$
\begin{aligned}
\delta(A) & =\Delta(I)=\operatorname{diam}_{d}(I) \leq \operatorname{diam}_{d}(A) \leq \operatorname{diam}_{d}(\hat{I} \cup \hat{J}) \\
& \leq \operatorname{diam}_{d}(\hat{I})+\operatorname{diam}_{d}(\hat{J})=\Delta(\hat{I})+\Delta(\hat{J}) \\
& \leq 2^{m}[\Delta(I)+\Delta(J)] \leq 2^{m+1} \Delta(I)=2^{m+1} \delta(A) .
\end{aligned}
$$

Thus it suffices to exhibit such $I$ and $J$.

Suppose $\mathcal{F} \subset \mathcal{J}$ is some family of $2^{m}$-adic arcs (e.g., defined by certain properties). We say that an arc $I^{n} \in \mathcal{J}^{n}$ is maximal with respect to $\mathcal{F}$ provided $I^{n} \in \mathcal{F}$ and for all $J^{l} \in \mathcal{J}^{l}$ with $J^{l} \in \mathcal{F}$, either $\Delta\left(J^{l}\right)<\Delta\left(I^{n}\right)$ or

$$
\Delta\left(J^{l}\right)=\Delta\left(I^{n}\right) \text { and } l \geq n .
$$

Thus $I^{n}$ is the "largest" arc in $\mathcal{F}$, and when there are several such large arcs, "seniority wins". Note that the parent of such a maximal $I^{n}$ will not belong to $\mathcal{F}$.

Now assume $A$ is the oriented arc $[a, b] \subset \mathrm{S}^{1}=[0,1] / \sim$ with $0<a<b<1$. Pick $I=I^{n} \in \mathcal{J}$ so that $I \subset A, \Delta(I)=\delta(A)$, and such that $I$ is maximal among all such arcs. Let $\hat{I} \supset I$ be the $\mathcal{J}$-parent of $I$. If $A \subset \hat{I}$, then upon setting $J:=I$ we are done.

Assume that $A \not \subset \hat{I}$. The maximality of $I$ ensures that one endpoint of $\hat{I}$, without loss of generality the left endpoint, is not contained in $A$. Let $y$ be the right endpoint of $\hat{I}$. Then $[a, y] \subset \hat{I}$. 
Now consider subarcs $J \in \mathcal{J}$ that lie in $A$ and to the right of $y$, and select the largest of these. More precisely, let $J=J^{l} \in \mathcal{J}$ be the maximal $2^{m}$-adic subarc that contains $y$ as its left endpoint and is contained in $[y, b]$. Note that the maximality of $I$ implies that

$$
\text { either } \quad l \geq n \quad \text { or } \quad \Delta(J)<\Delta(I) \text {. }
$$

Consider the parent $\hat{J}$ of $J$. We claim that $\hat{J}$ contains a point to the right of $b$, and then since $A=[a, y] \cup[y, b] \subset \hat{I} \cup \hat{J}$, we are done. If $\hat{J}$ did not contain a point to the right of $b$, then it would have to contain a point to the left of $y$, but as we now show this would lead to a contradiction.

So, suppose $\hat{J}$ contains a point to the left of $y$. Then in particular, $y$ is an interior point of $\hat{J}$. Since $y$ is an endpoint of $\hat{I}$, we cannot have $\hat{I} \supset \hat{J}$ or $\hat{I}=\hat{J}$, and therefore $\hat{I} \subsetneq \hat{J}$. This implies that $n>l$. However, it also implies that some $2^{m}$-adic sibling $\tilde{\tilde{J}}$ of $J$ satisfies $\tilde{J} \supset \hat{I}$, and therefore $\Delta(I) \leq \Delta(\hat{I}) \leq \Delta(\tilde{J})=\Delta(J)$. In view of (3.3), one of these last two implications does not hold, so $\hat{J}$ cannot contain a point to the left of $y$.

3.6. Proof of Lemma 3.4. An appeal to Lemma 2.2(b,c) permits us to assume that $(\Gamma,|\cdot|)$ is 1-bounded turning. Write $\Gamma[x, y]$ for the smaller diameter subarc joining points $x, y$ on $\Gamma$; so $|x-y|=\operatorname{diam}(\Gamma[x, y])$. Fix points $s, t$ on $S^{1}$ and put $x:=\varphi(s), y:=\varphi(t)$. Let $[s, t],[t, s]$ be the two arcs in $S^{1}$ joining $s, t$ and assume that $\operatorname{diam}_{d}([t, s]) \geq \operatorname{diam}_{d}([s, t])=d(s, t)$.

First we show that $|x-y| \leq 2^{m+1} K d(s, t)$. Using Lemma 3.5 we select $2^{m}$-adic $\operatorname{subarcs} I, J \in \mathcal{J}$ with $I \cup J \subset[s, t] \subset \hat{I} \cup \hat{J}, \hat{I} \cap \hat{J} \neq \emptyset$, and

$$
\Delta(J) \leq \Delta(I)=\delta([s, t]) \leq \operatorname{diam}_{d}([s, t])=d(s, t) .
$$

Here $\hat{I}, \hat{J} \in \mathcal{J}$ are the parents of $I, J$ relative to $\mathcal{J}$. Then

$$
\begin{aligned}
|x-y| & =\operatorname{diam}(\Gamma[x, y])=\min \{\operatorname{diam}(\varphi[s, t]), \operatorname{diam}(\varphi[t, s])\} \leq \operatorname{diam}(\varphi[s, t]) \\
& \leq \operatorname{diam}(\varphi(\hat{I} \cup \hat{J})) \leq K[\Delta(\hat{I})+\Delta(\hat{J})] \leq 2^{m} K[\Delta(I)+\Delta(J)] \\
& \leq 2^{m+1} K \Delta(I) \leq 2^{m+1} K d(s, t) .
\end{aligned}
$$

Next we show that $d(s, t) \leq 2^{m+1} K|x-y|$. Let $A$ be the subarc of $\mathrm{S}^{1}$ (either $A=[s, t]$ or $A=[t, s])$ with $\varphi(A)=\Gamma[x, y]$. Again we use Lemma 3.5 to pick a subarc $I \in \mathcal{J}$ with $I \subset A$ and $\Delta(I)=\delta(A)$. Then $\varphi(I) \subset \varphi(A)=\Gamma[x, y]$, so

$$
\begin{aligned}
d(s, t) & \leq \operatorname{diam}_{d}(A) \leq 2^{m+1} \delta(A)=2^{m+1} \Delta(I) \leq 2^{m+1} K \operatorname{diam}(\varphi(I)) \\
& \leq 2^{m+1} K \operatorname{diam}(\Gamma[x, y])=2^{m+1} K|x-y|
\end{aligned}
$$

We end this subsection with a criterion that describes when a metric circle in $\mathcal{S}_{1}$ is doubling. Roughly speaking, we get doubling if and only if diameters are always at least halved after a fixed number of steps. 
Lemma 3.7. Let $\mathcal{I} \stackrel{\Delta}{\rightarrow}(0,1]$ be a dyadic diameter function with snowflake parameter $\sigma=1$ and define $d:=d_{\Delta}$ as in (3.1). Then $\left(\mathrm{S}^{1}, d\right)$ is doubling if and only if there exists an $n_{0} \in \mathbb{N}$ such that

$$
\forall n \in \mathbb{N}, \forall I^{n}, \forall I^{n+n_{0}} \subset I^{n}, \quad \Delta\left(I^{n+n_{0}}\right) \leq \frac{1}{2} \Delta\left(I^{n}\right) .
$$

Proof. Suppose $\left(\mathrm{S}^{1}, d\right)$ is doubling. Then there are constants $C \geq 1$ and $\alpha \geq 1$ such that for each $r$-separated set $E$ in $\left(\mathrm{S}^{1}, d\right)$,

$$
\operatorname{card}(E) \leq C\left(\operatorname{diam}_{d}(E) / r\right)^{\alpha} .
$$

Let $I:=I^{n} \in \mathcal{I}^{n}$ be given. Suppose $\left(I^{m}\right)_{m=n}^{n+k}$ is a descendant sequence with $\Delta\left(I^{m}\right) \geq r:=\frac{1}{2} \Delta(I)$ for all $m \in\{n, n+1, \ldots, n+k\}$. Let $E$ be the set of endpoints of all the subarcs $I^{n}, \ldots, I^{n+k}$. To see that $E$ is $r$-separated, let $e_{1}, e_{2}$ be two distinct points in $E$. We can assume that $e_{1}$ is an endpoint of some $I^{i}$ and $e_{2} \in I^{j} \subset I^{i}$, where $n \leq i<j \leq n+k$, and that $I^{j}$ does not contain $e_{1}$ but $I^{j-1}$ does. Then the sibling $\tilde{I}^{j}$ of $I^{j}$ separates $e_{1}$ and $e_{2}$. Thus $d\left(e_{1}, e_{2}\right) \geq$ $\Delta\left(\tilde{I}^{j}\right)=\Delta\left(I^{j}\right) \geq r$.

Now $\operatorname{diam}_{d}(E)=\operatorname{diam}_{d}(I)=\Delta(I)=2 r$, so by doubling,

$$
k \leq \operatorname{card}(E) \leq C\left(\operatorname{diam}_{d}(E) / r\right)^{\alpha}=2^{\alpha} C .
$$

Therefore $n_{0}:=\left\lceil 2^{\alpha} C\right\rceil+1$ is the desired number.

Conversely, suppose there is such an $n_{0} \in \mathbb{N}$. Let $A \subset \mathrm{S}^{1}$ be any arc. Let $I \in \mathcal{I}^{n}$, $J \in \mathcal{I}^{m}$ be dyadic subarcs with parents $\hat{I} \in \mathcal{I}^{n-1}, \hat{J} \in \mathcal{I}^{m-1}$ as in Lemma 3.5; thus $I \cup J \subset A \subset \hat{I} \cup \hat{J}$. Let $I_{1}, \ldots, I_{2^{n_{0}+1}} \in \mathcal{I}^{n+n_{0}}, J_{1}, \ldots, J_{2^{n_{0}+1}} \in \mathcal{I}^{m+n_{0}}$ be the dyadic subarcs contained in $\hat{I}$ and $\hat{J}$ respectively. Then for all $1 \leq k \leq 2^{n_{0}+1}$

$$
\operatorname{diam}_{d}\left(I_{k}\right)=\Delta\left(I_{k}\right) \leq \frac{1}{2} \operatorname{diam}_{d}(I) \leq \operatorname{diam}_{d}(A)
$$

and similarly $\operatorname{diam}_{d}\left(J_{k}\right) \leq(1 / 2) \operatorname{diam}_{d}(A)$. Thus we obtain the doubling condition with $N:=2^{n_{0}+2}$.

\section{Proof of the Main Theorem}

Here we establish parts (A), (B), (C) of the theorem stated in the introduction; see $\S 4.1, \S 4.2$ and $\S 4.3$, respectively. In addition, in $\S 4.3$ we explain how to recover Rohde's Theorem.

Recall from $\S 3.2$ that $\mathcal{S}_{\sigma}$ is the collection of all metric circles $\left(\mathrm{S}^{1}, d_{\sigma}\right)$ where the metrics $d_{\sigma}=d_{\Delta}$ are defined as in $(3.1)$ and $\Delta: \mathcal{I} \rightarrow(0,1]$ is any dyadic diameter function constructed using the snowflake parameter $\sigma \in[1 / 2,1]$. Recall too that for $\sigma \in[1 / 2,1)$ each curve in $\mathcal{S}_{\sigma}$ is a metric quasicircle that has Assouad dimension at most $\log 2 / \log (1 / \sigma)$; see Lemma $3.1(\mathrm{c}, \mathrm{e})$. 
For the remainder of this section, $(\Gamma,|\cdot|)$ is a bounded turning circle. Our three proofs share the following common theme: We define an appropriate shrinking subdivision for $\Gamma$ and then appeal to Proposition 2.4 and Lemma 3.4 to obtain the necessary bi-Lipschitz homeomorphisms. In each case this involves constructing a dyadic diameter function $\Delta$ using some snowflake parameter.

To start, we fix an orientation on $\Gamma$. All subarcs inherit this orientation, and $[a, b]$ denotes the oriented subarc of $\Gamma$ with endpoints $a, b$. Next, an appeal to Lemma $2.2(\mathrm{~b}, \mathrm{c})$ permits us to replace $|\cdot|$ with its associated diameter distance thereby obtaining a bi-Lipschitz equivalent 1-bounded turning circle; the bi-Lipschitz constant for this change of metric equals the original bounded turning constant. Thus we may, and do, assume that $(\Gamma,|\cdot|)$ is 1 -bounded turning. This means that

$$
\operatorname{diam}([a, b])=|a-b| \quad \text { whenever } \quad \operatorname{diam}([a, b]) \leq \operatorname{diam}(\Gamma \backslash[a, b]) .
$$

We also assume that $\operatorname{diam}(\Gamma)=1$; this involves another bi-Lipschitz change of metric with bi-Lipschitz constant $\max \left\{\operatorname{diam}(\Gamma), \operatorname{diam}(\Gamma)^{-1}\right\}$.

\subsection{Proof of (A)}

We assume $(\Gamma,|\cdot|)$ is 1-bounded turning with $\operatorname{diam}(\Gamma)=1$; it need not be doubling. We construct a dyadic diameter function $\Delta$ on $\mathcal{I}$, using the snowflake parameter $\sigma=1$, so that $(\Gamma,|\cdot|)$ is bi-Lipschitz equivalent to $\left(\mathrm{S}, d_{\Delta}\right)$.

First, we divide $\Gamma$ into two arcs $A_{0}^{1}, A_{1}^{1}$ that both have diameter one. Then we inductively divide each arc into two subarcs of equal diameter. Appealing to Proposition 2.3, we divide each $A_{i}^{n}$ into two subarcs $A_{2 i}^{n+1}, A_{2 i+1}^{n+1}$ of equal diameter. This defines subarcs $A_{k}^{n}$ for each $k \in\left\{0,1, \ldots, 2^{n}-1\right\}$ and all $n \in \mathbb{N}$. Here we label so that the $A_{k}^{n}$ are ordered successively along $\Gamma$ with the initial point of $A_{0}^{n}$ the same for all $n \in \mathbb{N}$.

We claim that $\lim _{n \rightarrow \infty} \max _{k} \operatorname{diam}\left(A_{k}^{n}\right)=0$. Suppose this does not hold. Then there is an $\varepsilon>0$ such that the set $\boldsymbol{\Gamma}_{\varepsilon}:=\left\{A_{k}^{n} \mid \operatorname{diam}\left(A_{k}^{n}\right) \geq \varepsilon\right\}$ is infinite. Noting that each parent of an arc in $\boldsymbol{\Gamma}_{\varepsilon}$ also belongs to $\boldsymbol{\Gamma}_{\varepsilon}$, we may appeal to König's Lemma to obtain a descendent sequence $\mathrm{S}^{1}=: A^{0} \supset A^{1} \supset A^{2} \supset \cdots$ (where $A^{n}=A_{k_{n}}^{n}$ is some arc in $\boldsymbol{\Gamma}_{\varepsilon}$ ). By construction $A^{n}$ is divided into two subarcs $A^{n+1}$ and $B^{n+1}$ of equal diameter, so $\operatorname{diam}\left(B^{n+1}\right) \geq \varepsilon$. Then $\left\{B^{1}, B^{2}, \ldots\right\}$ is an infinite collection of non-overlapping subarcs of $\Gamma$ each with diameter at least $\varepsilon$. This contradiction to Lemma 2.1 implies that our claim must hold

By setting $\mathcal{A}^{n}:=\left\{A_{k}^{n} \mid k \in\left\{0,1, \ldots, 2^{n}-1\right\}\right\}$ (for each $n \in \mathbb{N}$ ) we obtain a shrinking subdivision $\left(\mathcal{A}^{n}\right)_{1}^{\infty}$ for $\Gamma$; see $\S 2.6$. In fact, $\left(\mathcal{I}^{n}\right)_{1}^{\infty}$ and $\left(\mathcal{A}^{n}\right)_{1}^{\infty}$ are combinatorially equivalent shrinking subdivisions, and thus by Proposition 2.4 there is an induced homeomorphism $\varphi: \mathrm{S}^{1} \rightarrow \Gamma$ with $\varphi\left(I_{k}^{n}\right)=A_{k}^{n}$ for all $n \in \mathbb{N}$ and all $k \in\left\{0,1, \ldots, 2^{n}-1\right\}$.

It remains to construct a dyadic diameter function $\Delta$ using the snowflake parameter $\sigma=1$ and so that $\Delta$ also satisfies the following: for all $n \in \mathbb{N}$,

$$
\text { for all } k \in\left\{0,1, \ldots, 2^{n}-1\right\}, \quad \frac{1}{2} \Delta\left(I_{k}^{n}\right) \leq \operatorname{diam}\left(A_{k}^{n}\right) \leq 2 \Delta\left(I_{k}^{n}\right) .
$$


Having accomplished this task, we can appeal to Lemma 3.4 (with $C=1, m=1$, and $K=2)$ to assert that $\varphi:\left(\mathrm{S}^{1}, d_{\Delta}\right) \rightarrow(\Gamma,|\cdot|)$ is 8-bi-Lipschitz.

We start by setting $\Delta\left(\mathrm{S}^{1}\right)=\Delta\left(I_{0}^{1}\right)=\Delta\left(I_{1}^{1}\right):=1$ and note that (4.1) holds for $n=1$. Now assume that for some $n \in \mathbb{N}$ and all $k \in\left\{0,1, \ldots, 2^{n}-1\right\}, \Delta\left(I_{k}^{n}\right)$ has been defined so that (4.1) holds. Consider a dyadic subarc $I^{n}=I_{k}^{n}$, its two children $I^{n+1}, \tilde{I}^{n+1} \subset I^{n}$, and its corresponding $\operatorname{arc} A^{n}=A_{k}^{n}=\varphi\left(I_{k}^{n}\right) \subset \Gamma$. We note that by construction each child $A^{n+1}$ of $A^{n}$ satisfies

$$
\frac{1}{2} \operatorname{diam}\left(A^{n}\right) \leq \operatorname{diam}\left(A^{n+1}\right) \leq \operatorname{diam}\left(A^{n}\right) .
$$

We examine two cases. If $\Delta\left(I^{n}\right) \leq \operatorname{diam}\left(A^{n}\right)$, then we define

$$
\Delta\left(I^{n+1}\right)=\Delta\left(\tilde{I}^{n+1}\right):=\Delta\left(I^{n}\right) .
$$

We see that (4.1) holds (for $n+1$ ), since

$$
\begin{aligned}
\frac{1}{2} \Delta\left(I^{n+1}\right) & =\frac{1}{2} \Delta\left(I^{n}\right) \leq \frac{1}{2} \operatorname{diam}\left(A^{n}\right) \leq \operatorname{diam}\left(A^{n+1}\right) \\
& \leq \operatorname{diam}\left(A^{n}\right) \leq 2 \Delta\left(I^{n}\right)=2 \Delta\left(I^{n+1}\right) .
\end{aligned}
$$

Here (4.1) was used for $n$ in the last inequality.

If $\Delta\left(I^{n}\right)>\operatorname{diam}\left(A^{n}\right)$, then we define

$$
\Delta\left(I^{n+1}\right)=\Delta\left(\tilde{I}^{n+1}\right):=\frac{1}{2} \Delta\left(I^{n}\right) .
$$

Again one checks that (4.1) holds (for $n+1$ ), since

$$
\begin{aligned}
\frac{1}{2} \Delta\left(I^{n+1}\right) & =\frac{1}{4} \Delta\left(I^{n}\right) \leq \frac{1}{2} \operatorname{diam}\left(A^{n}\right) \leq \operatorname{diam}\left(A^{n+1}\right) \\
& \leq \operatorname{diam}\left(A^{n}\right)<\Delta\left(I^{n}\right)=2 \Delta\left(I^{n+1}\right) .
\end{aligned}
$$

Here (4.1) was used for $n$ in the first inequality.

\subsection{Proof of $(B)$}

We assume $(\Gamma,|\cdot|)$ is 1-bounded turning with $\operatorname{diam}(\Gamma)=1$ and doubling with finite Assouad dimension $\alpha$. Fix any $\sigma \in\left(2^{-1 / \alpha}, 1\right)$ (equivalently, $\alpha<\log 2 / \log (1 / \sigma)$ ). We construct a dyadic diameter function $\Delta$ on $\mathcal{I}$, using the snowflake parameter $\sigma$, so that $(\Gamma,|\cdot|)$ is bi-Lipschitz equivalent to $\left(\mathrm{S}, d_{\Delta}\right)$. In contrast to our above proof of (A), here we do " $m$ steps at the same time"; i.e., each arc will be divided into $2^{m}$ subarcs of the same diameter. That is, we will in fact construct a $2^{m}$-adic diameter function; see $\S 3.3$.

Put $\beta:=\log 2 / \log (1 / \sigma)$, so $\sigma=2^{-1 / \beta}$. Then since $\beta>\alpha=\operatorname{dim}_{\mathcal{A}}(\Gamma)$, there exists an $\varepsilon_{0} \in(0,1]$ such that for all $\varepsilon \in\left(0, \varepsilon_{0}\right)$, the cardinality of any $\varepsilon D$-separated set $S \subset \Gamma$ with $D=\operatorname{diam}(S)$ satisfies

$$
\operatorname{card}(S)<\varepsilon^{-\beta}
$$


Since $\sigma=2^{-1 / \beta}<1$, we may select an $m \in \mathbb{N}$ so that

$$
\tau:=\sigma^{m}=\left(2^{-1 / \beta}\right)^{m}=\left(2^{m}\right)^{-1 / \beta}<\varepsilon_{0} .
$$

In particular, if $S$ is a $\tau D$-separated subset of $\Gamma$, with $D=\operatorname{diam}(S)$, then $\operatorname{card}(S)<$ $\tau^{-\beta}=2^{m}:=M$.

It now follows that whenever we divide an $\operatorname{arc} A$ of $\Gamma$ into $M$ subarcs $A_{k}$ all with equal diameters, then

$$
M^{-1} \operatorname{diam}(A) \leq \operatorname{diam} A_{k} \leq \tau \operatorname{diam}(A) .
$$

The lefthand inequality follows directly from the triangle inequality whereas the righthand inequality holds because there are at least $M$ distinct endpoints of the subarcs $A_{k}$ (which are separated by $\operatorname{diam} A_{k}$ ) and so, by the above, these endpoints cannot be $\tau D$-separated with $D:=\operatorname{diam}(A)$

We use Proposition 2.3 to divide $\Gamma$ into $M \operatorname{arcs} A_{0}^{1}, A_{1}^{1}, \ldots, A_{M-1}^{1}$, all of equal diameter. We iterate this procedure: assuming that $\operatorname{arcs} A_{k}^{n}\left(k \in\left\{0,1, \ldots, M^{n}-1\right\}\right)$ have been so constructed, each arc $A_{k}^{n}$ is subdivided into $M$ subarcs $A_{k M+j}^{n+1}$ (with $j \in\{0,1, \ldots, M-1\})$ all of equal diameter; the subarcs $A_{k M+j}^{n+1}$ are labeled successively along $A_{k}^{n}$. To avoid possible confusion, we note that all subarcs of the same $\operatorname{arc} A_{k}^{n}$ have the same diameters, however, subarcs of different $\operatorname{arcs} A_{i}^{n}, A_{j}^{n}$ do not necessarily have the same diameters.

Let $\mathcal{J}=\bigcup_{n=0}^{\infty} \mathcal{J}^{n}$ be the family of all $M$-adic subarcs of $\mathrm{S}^{1}$; here $M=2^{m}$ and $\mathcal{J}^{n}=\mathcal{I}^{m n}$ consists of the $M^{n}=2^{m n}$ subarcs of the form $J_{k}^{n}:=\left[k / 2^{m n}\right.$, $\left.(k+1) / 2^{m n}\right] \in \mathcal{I}^{m n}$ with $k \in\left\{0,1, \ldots, 2^{m n}-1\right\}$. See the last paragraph of $\S 3.1$.

Setting $\mathcal{A}^{n}:=\left\{A_{k}^{n} \mid k \in\left\{0,1, \ldots, M^{n}-1\right\}\right\}$ (for each $n \in \mathbb{N}$ ) we obtain a shrinking subdivision $\left(\mathcal{A}^{n}\right)_{1}^{\infty}$ for $\Gamma$; see $\S 2.6$. In fact, $\left(\mathcal{J}^{n}\right)_{1}^{\infty}$ and $\left(\mathcal{A}^{n}\right)_{1}^{\infty}$ are combinatorially equivalent shrinking subdivisions, and thus by Proposition 2.4 there is an induced homeomorphism $\varphi: \mathrm{S}^{1} \rightarrow \Gamma$ with $\varphi\left(J_{k}^{n}\right)=A_{k}^{n}$ for all $n \in \mathbb{N}$ and all $k \in\left\{0,1, \ldots, M^{n}-1\right\}$.

Now we construct an $M$-adic diameter function $\mathcal{J} \stackrel{\Delta}{\rightarrow}(0,1]$ using the snowflake parameter $\tau$ and so that $\Delta$ also satisfies the following: for all $n \in \mathbb{N}$ and for all $k \in\left\{0,1, \ldots, M^{n}-1\right\}$,

$$
K^{-1} \Delta\left(J_{k}^{n}\right) \leq \operatorname{diam}\left(A_{k}^{n}\right) \leq K \Delta\left(J_{k}^{n}\right),
$$

where $K:=\tau M$. Once this task is completed, we can appeal to Lemma 3.4 (with $C=1$ and $\left.2^{m}=M\right)$ to assert that $\varphi:\left(\mathrm{S}^{1}, d_{\Delta}\right) \rightarrow(\Gamma,|\cdot|)$ is $L$-bi-Lipschitz with $L=2 M K=2 \tau M^{2}$.

To start, we set $\Delta\left(\mathrm{S}^{1}\right):=1$ and then for each $k \in\{0,1, \ldots, M-1\}$, we put $\Delta\left(J_{k}^{1}\right):=\tau$. To check (4.4) for $n=1$ we use (4.3) and the fact that $\operatorname{diam}(\Gamma)=1$ to see that

$$
\frac{1}{K} \Delta\left(J_{k}^{1}\right)=\frac{\tau}{K}=\frac{1}{M} \leq \operatorname{diam}\left(A_{k}^{1}\right) \leq \tau=\Delta\left(J_{k}^{1}\right) .
$$

Assume that for some $n \in \mathbb{N}$ and all $k \in\left\{0,1, \ldots, M^{n}-1\right\}, \Delta\left(J_{k}^{n}\right)$ has been defined so that (4.4) holds. Fix any $M$-adic subarc $J^{n}=J_{k}^{n}$ and let $A^{n}=A_{k}^{n}=\varphi\left(J_{k}^{n}\right)$ be the corresponding subarc of $\Gamma$. We consider two cases. 
First, suppose that $\Delta\left(J^{n}\right) \leq \operatorname{diam}\left(A^{n}\right)$. Then we define the diameter of each child $J^{n+1}$ of $J^{n}$ by

$$
\Delta\left(J^{n+1}\right):=\tau \Delta\left(J^{n}\right) .
$$

To confirm that (4.4) is still satisfied for all these children, we observe that

$$
\begin{aligned}
\frac{1}{K} \Delta\left(J^{n+1}\right) & =\frac{1}{M} \Delta\left(J^{n}\right) \leq \frac{1}{M} \operatorname{diam}\left(A^{n}\right) \leq \operatorname{diam}\left(A^{n+1}\right) \\
& \leq \tau \operatorname{diam}\left(A^{n}\right) \leq \tau K \Delta\left(J^{n}\right)=K \Delta\left(J^{n+1}\right) .
\end{aligned}
$$

Here the initial inequality holds by supposition, the next two inequalities follow from (4.3), and the induction hypothesis gives the last inequality.

Next, suppose that $\Delta\left(J^{n}\right)>\operatorname{diam}\left(A^{n}\right)$. Now we define the diameter of each child $J^{n+1}$ of $J^{n}$ by

$$
\Delta\left(J^{n+1}\right):=\frac{1}{M} \Delta\left(J^{n}\right)=\frac{1}{2^{m}} \Delta\left(J^{n}\right) .
$$

To check that (4.4) holds for all these children, we again observe that

$$
\begin{aligned}
\frac{1}{K} \Delta\left(J^{n+1}\right) & =\frac{1}{K M} \Delta\left(J^{n}\right) \leq \frac{1}{M} \operatorname{diam}\left(A^{n}\right) \leq \operatorname{diam}\left(A^{n+1}\right) \\
& \leq \tau \operatorname{diam}\left(A^{n}\right) \leq \tau \Delta\left(J^{n}\right)=K \Delta\left(J^{n+1}\right) .
\end{aligned}
$$

Here the initial inequality holds by the induction hypothesis, the next two inequalities follow from (4.3), and our supposition gives the last inequality.

This finishes the construction of an $M$-adic diameter function $\Delta$ for which (4.4) holds for all $n \in \mathbb{N}$ and all $k \in\left\{0,1, \ldots, M^{n}-1\right\}$.

Having defined an appropriate $M$-adic diameter function $\Delta$ on $\mathcal{J}$, we use Lemma 3.4 to deduce that $\varphi:\left(\mathrm{S}^{1}, d_{\tau}\right) \rightarrow(\Gamma,|\cdot|)$ is $L$-bi-Lipschitz, where $d_{\tau}:=d_{\Delta}$. The $M$-adic diameter function $\Delta$, constructed using the snowflake parameter $\tau$, can be extended to a dyadic diameter function $\Delta$ that is constructed with the snowflake parameter $\sigma=\tau^{1 / m}$. See the discussion in $\S 3.3$. Let $d_{\sigma}$ be the metric associated with the dyadic diameter function $\Delta$. According to Lemma 3.2, the identity map id : $\left(\mathrm{S}^{1}, d_{\sigma}\right) \rightarrow\left(\mathrm{S}^{1}, d_{\tau}\right)$ is $M$-bi-Lipschitz. It now follows that $(\Gamma,|\cdot|)$ is $(M L)$-bi-Lipschitz equivalent to the metric quasicircle $\left(\mathrm{S}^{1}, d_{\sigma}\right) \in \mathcal{S}_{\sigma}$.

Remark 4.1. We can easily adjust the previous proof to obtain a model circle constructed from a 4-adic diameter function. To do so, we choose $m$ in (4.2) to be even; say, $m=2 k$, so $M=4^{k}$. Then we extend the $M$-adic diameter function $\mathcal{J} \rightarrow(0,1]$ to a 4 -adic diameter function with snowflake parameter $p:=\tau^{1 / k}=$ $\sigma^{2} \in\left(4^{-1 / \alpha}, 1\right)$ as described in Remark 3.3. This yields a metric $d$, constructed via the 4 -adic diameter function, such that the original metric quasicircle $(\Gamma,|\cdot|)$ is bi-Lipschitz equivalent to $\left(\mathrm{S}^{1}, d\right)$. Thus the following variant of $(\mathrm{B})$ holds.

Corollary $4.2\left(\left(\mathrm{~B}^{\prime}\right)\right)$. Let $(\Gamma,|\cdot|)$ be a metric quasicircle with finite Assouad dimension $\alpha$. Then for each $p \in\left(4^{-1 / \alpha}, 1\right)$ there is a 4-adic diameter function $\Delta$, constructed with snowflake parameter $p$, and an associated metric $d=d_{\Delta}$, such that $\left(\mathrm{S}^{1}, d\right)$ is bi-Lipschitz equivalent to $(\Gamma,|\cdot|)$. 
Note that $1 \leq \alpha<2$ is equivalent to $1 / 4 \leq 4^{-1 / \alpha}<1 / 2$, so in this case we can choose $p \in\left(4^{-1 / \alpha}, 1 / 2\right) \subset(1 / 4,1 / 2)$.

\subsection{Planar quasicircles}

In Paragraph 4.3 below we prove part (C) of our theorem. Then we explain how to recover Rohde's theorem. We begin with a precise description for the construction of Rohde snowflakes that includes some useful geometric estimates.

Everywhere throughout this subsection $\mathcal{J}$ denotes the family of 4 -adic subarcs of the circle $\mathrm{S}^{1}$.

Each Rohde snowflake $R$, constructed using a parameter $p \in[1 / 4,1 / 2)$, is the Hausdorff limit of a sequence $\left(R^{n}\right)_{1}^{\infty}$ of polygons where $R^{n+1}$ is obtained from $R^{n}$ by using the replacement choices illustrated in Figure 1. Both the snowflake parameter $p$ and the polygonal arc $A_{p}$ are kept fixed throughout the construction.

We start with the unit square $R^{1}=E_{0}^{1} \cup E_{1}^{1} \cup E_{2}^{1} \cup E_{3}^{1}$, so each $E_{k}^{1}$ is a Euclidean line segment of diameter one and these are labeled successively along $R^{1}$. Suppose we have constructed $R^{n}$ as a union of $4^{n}$ Euclidean line segments $E_{k}^{n}$, $k \in\left\{0,1, \ldots, 4^{n}-1\right\}$ (labeled successively along $R^{n}$ ). Then for each of the edges $E_{k}^{n}$ of $R^{n}$ we have two choices: either we replace $E_{k}^{n}$ with the four line segments obtained by dividing $E_{k}^{n}$ into four segments of equal diameter, or we replace $E_{k}^{n}$ by a similarity copy of the polygonal arc $A_{p}$ pictured at the top right of Figure 1 . In both cases $E_{k}^{n}$ is replaced by four new line segments $E_{4 k+j}^{n+1}$ (with $j \in\{0,1,2,3\}$ ) that we call the children of $E_{k}^{n}$, so $E_{k}^{n}$ is the parent of each of $E_{4 k}^{n+1}, E_{4 k+1}^{n+1}, E_{4 k+2}^{n+1}, E_{4 k+3}^{n+1}$. Each of these children has Euclidean diameter equal to either $(1 / 4) \operatorname{diam}\left(E_{k}^{n}\right)$ in the first case or $p \operatorname{diam}\left(E_{k}^{n}\right)$ in the second case. The second type of replacement is done so that the "tip" of the replacement arc points into the exterior of $R^{n}$. Then $R^{n+1}$ is the union of the $4^{n+1} \operatorname{arcs} E_{i}^{n+1}$ (with $i \in\left\{0,1, \ldots, 4^{n+1}-1\right\}$ ).

We call the line segments $E_{k}^{n}$ the 4 -adic edges of $R^{n}$. We note that different replacement rules can be used for different edges $E_{i}^{n}, E_{j}^{n}$ of $R^{n}$. Thus, for example, one edge could have diameter $1 / 4^{n}$ while an adjacent edge might have diameter $p^{n}$ (which could be much larger). In any event, for each $n \in \mathbb{N}$ there is a natural homeomorphism $\varphi_{n}: \mathrm{S}^{1} \rightarrow R^{n}$ that is given by mapping each 4-adic subarc $J_{k}^{n} \subset S^{1}$ to the 4-adic edge $E_{k}^{n} \subset R^{n}$. We say that the edge $E_{k}^{n}$ corresponds to the subarc $J_{k}^{n}$.

Set $\theta=\theta(p):=2 \arcsin \left((2 p)^{-1}-1\right)$; this is the interior angle at the "tip" of the $\operatorname{arc} A_{p}$ in Figure 1, but see also the left-most picture in Figure 2. Also, notice that if $A_{p}$ is normalized to have diameter one, then its height is $(p-1 / 4)^{1 / 2}$.

Let $E$ be one of the 4 -adic edges of some $R^{n}$. We write $T(E)=T_{p}(E)$ for the closed isosceles triangle with base $E$ and height $\operatorname{diam}(E)(p-1 / 4)^{1 / 2}$; we orient $T(E)$ so that it "points" into the exterior of the polygon $R^{n}$. Thus if $E$ were to be replaced by a similarity copy of the arc $A_{p}$, then $T(E)$ would be the closed convex hull of this affine copy of $A_{p}$ (see the left-most picture in Figure 2) and the third vertex of $T(E)$ would correspond to the "tip" of this image of $A_{p}$. We call this third vertex the "tip" of $T(E)$. 


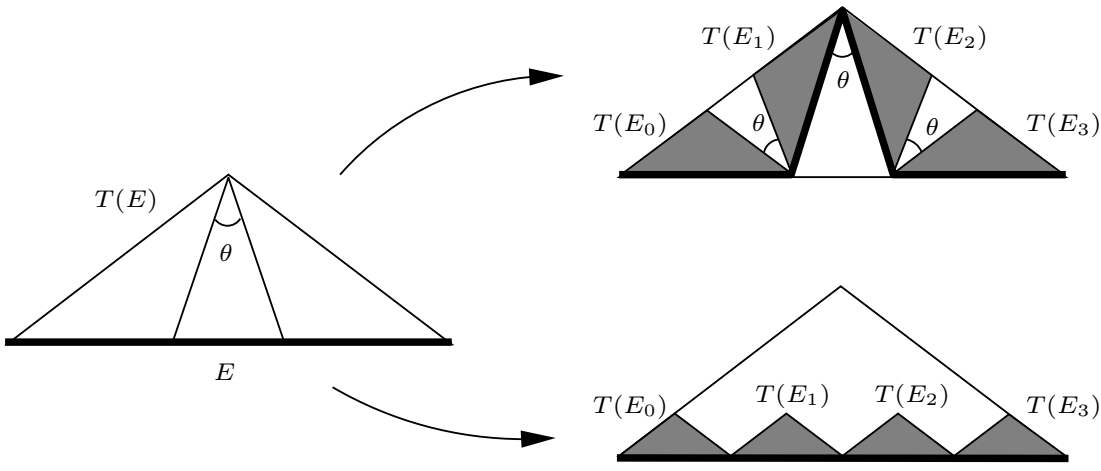

Figure 2. Triangles enclosing an arc.

Next, let $E_{0}, E_{1}, E_{2}, E_{3}$ be the four children of $E$. Not only are these children contained in $T(E)$, but elementary geometric considerations reveal that the associated triangles $T\left(E_{0}\right), T\left(E_{1}\right), T\left(E_{2}\right), T\left(E_{3}\right)$ are also contained in $T(E)$. See the two right-most pictures in Figure 2. A standard argument now reveals that the sequence $\left(\varphi_{n}\right)_{1}^{\infty}$ is uniformly Cauchy, and hence it converges to a continuous surjection $\varphi: \mathrm{S}^{1} \rightarrow R$ and the planar curve $R$ is the Hausdorff limit of the sequence $\left(R^{n}\right)_{1}^{\infty}$.

Consider a subcurve $A:=\varphi(J)$ of $R$ where $J$ is some 4 -adic subarc of $\mathrm{S}^{1}$. Let $E$ be the 4-adic edge that corresponds to $J$. We see that $A$ is "built on top of $E$ " in the sense that the replacement choices used to construct $R$, applied to the edge $E$, produce $A$. We write $A:=R(E)$ and call $A$ the 4-adic subarc of $R$ corresponding to $E$ (and to $J$ ). (This abuse of notation will be justified below - see (4.7), where we prove that $\varphi$ is injective, hence a homeomorphism, so $R$ is a Jordan curve and $A$ is an arc.) By induction, we deduce that $A$ also lies in $T(E)$ and has the same endpoints as $E$, therefore

$$
\operatorname{diam}(A)=\operatorname{diam}(T(E))=\operatorname{diam}(E) .
$$

Looking again at the right-most pictures in Figure 2, and appealing to elementary geometric considerations, we see that the angle between any pair of consecutive triangles $T\left(E_{0}\right), \ldots, T\left(E_{3}\right)$ is at least $\theta$. It is also elementary to check that

$$
\begin{aligned}
\operatorname{dist}\left(T\left(E_{0}\right), T\left(E_{3}\right)\right) & \geq \operatorname{dist}\left(T\left(E_{1}\right), T\left(E_{3}\right)\right) \\
& =\operatorname{dist}\left(T\left(E_{0}\right), T\left(E_{2}\right)\right) \geq c(p) \operatorname{diam}(E),
\end{aligned}
$$

where $c(p):=\frac{1}{2}-p$.

As final preparation for our proof of part (C), suppose $\hat{I}, \hat{J}$ are two adjacent 4-adic subarcs of $\mathrm{S}^{1}$, say with $\hat{I} \cap \hat{J}=\{\xi\}$. (These arcs might be from different generations; i.e., possibly $\hat{I}=J_{k}^{n}$ and $\hat{J}=J_{\ell}^{m}$ where $n \neq m$.) Let $\hat{E}, \hat{F}$ be the corresponding 4-adic edges, so $\hat{E} \cap \hat{F}=\{a\}$ where $a:=\varphi(\xi)$.

It follows from the above remarks that the angle between the two triangles $T(\hat{E})$ and $T(\hat{F})$, at their common vertex $a$, is at least $\theta$. See Figure 3. More 


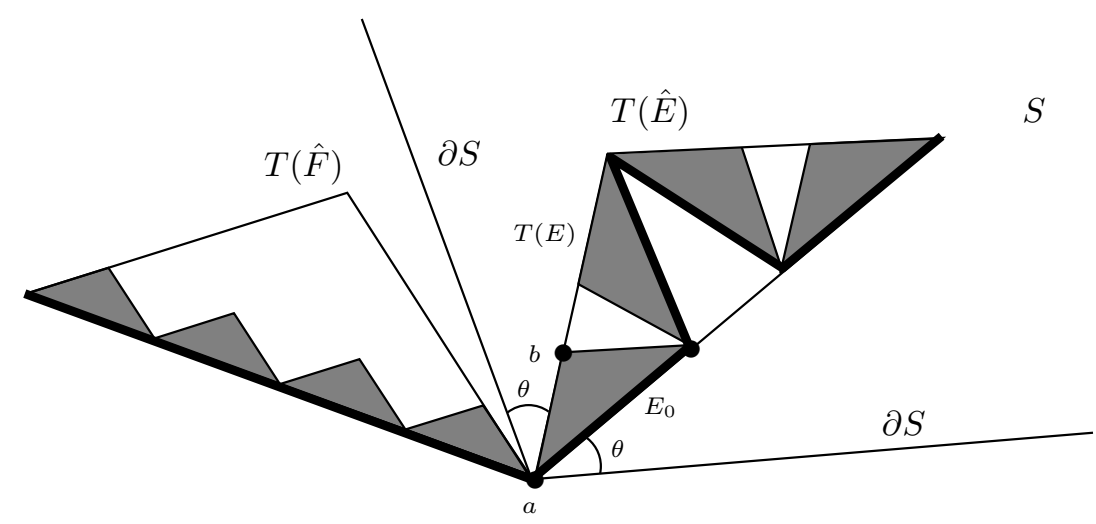

FiguRE 3. Separating points.

precisely, let $S$ be the closed sector, with vertex at $a$, that contains $T(\hat{E})$ and is such that $\theta$ is the angle between each edge of $\partial S$ and the nearest edge of $T(\hat{E})$. Then $T(\hat{F})$ lies in the closure of $\mathbb{R}^{2} \backslash S$.

Now suppose there is a child $E$ of $\hat{E}$ that does not contain $a$. Then $T(E)$ is compactly contained in the sector $S$ and in fact

$$
\operatorname{dist}(T(E), T(\hat{F})) \geq \operatorname{dist}(T(E), \partial S) \geq c(p) \operatorname{diam}(E),
$$

where again $c(p):=\frac{1}{2}-p$. This follows from the estimates

$$
\operatorname{dist}(T(E), \partial S) \geq \operatorname{dist}(b, \partial S) \geq c(p) \operatorname{diam}(E),
$$

where $b$ is the "tip" of the appropriate $T\left(E_{0}\right)$ as pictured in Figure 3.

Finally, fix points $s, t \in \hat{I} \cup \hat{J}$. Suppose there is a child $I$ of $\hat{I}$ whose interior, $\operatorname{int}(I)$, separates $s, t$ in $\hat{I} \cup \hat{J}$ (meaning that $s, t$ lie in different components of $(\hat{I} \cup \hat{J}) \backslash \operatorname{int}(I))$. We claim that

$$
|\varphi(s)-\varphi(t)| \geq c(p) \operatorname{diam}(\varphi(I)) .
$$

This follows from $(4.5)$ if both $\varphi(s), \varphi(t)$ lie in $T(\hat{E})$; otherwise it follows from (4.6). Also, see Figure 3.

Notice that injectivity of $\varphi$ follows from (4.7).

Having established the above terminology and geometric estimates, we now turn to the following:

4.3. Proof of (C). We use the notation and terminology introduced above.

It is well-known that planar quasicircles have Assouad dimension strictly less than two; see Lemma 4.1 in [16] or Theorem 5.2 in [11]. Furthermore, Assouad dimension is unchanged by bi-Lipschitz maps. Thus every metric quasicircle that is bi-Lipschitz equivalent to a planar quasicircle has Assouad dimension strictly less than two. 
Let $(\Gamma,|\cdot|)$ be a metric quasicircle with Assouad dimension $\alpha \in[1,2)$. We prove that $(\Gamma,|\cdot|)$ is bi-Lipschitz equivalent to a planar quasicircle. In fact, we show that it is bi-Lipschitz equivalent to a Rohde snowflake.

Fix $p \in\left(4^{-1 / \alpha}, 1 / 2\right) \subset(1 / 4,1 / 2)$. According to part (B) of our Theorem (more precisely, the version $\left(\mathrm{B}^{\prime}\right)$ stated as Corollary 4.2 ), there is a 4-adic diameter function $\Delta$ with snowflake parameter $p$ and associated metric $d_{p}$ such that $(\Gamma,|\cdot|)$ is bi-Lipschitz equivalent to $\left(\mathrm{S}^{1}, d_{p}\right)$.

We use the 4 -adic diameter function $\Delta$ to construct a Rohde snowflake $R$ with snowflake parameter $p$, and we prove that $\left(\mathrm{S}^{1}, d_{p}\right)$ is bi-Lipschitz equivalent to $R$. Hence $(\Gamma,|\cdot|)$ is bi-Lipschitz equivalent to a planar quasicircle.

Recall that $\mathcal{J}$ is the set of all 4 -adic subarcs of $\mathrm{S}^{1}$; similarly, $\mathcal{J}^{n}:=\mathcal{I}^{4 n}$.

It is convenient to scale the metric $d_{p}$-so also the diameter function $\Delta$ - by the factor $1 / p$. This bi-Lipschitz change in our metric means that for each $J_{k}^{1} \in \mathcal{J}^{1}$, $\Delta\left(J_{k}^{1}\right)=1$. See the paragraph immediately following (4.4).

The desired Rohde snowflake $R$ is the limit of a sequence $\left(R^{n}\right)_{1}^{\infty}$ of polygons, and we must describe how to replace each edge of $R^{n}$ to obtain $R^{n+1}$. Of course, we start with the unit square $R^{1}:=E_{0}^{1} \cup E_{1}^{1} \cup E_{2}^{1} \cup E_{3}^{1}$, so each edge $E_{k}^{1}$ satisfies $\Delta\left(J_{k}^{1}\right)=$ $1=\operatorname{diam}\left(E_{k}^{1}\right)$. Now suppose that we have constructed polygons $R^{1}, R^{2}, \ldots, R^{n}:=$ $E_{0}^{n} \cup \cdots \cup E_{4^{n}-1}^{n}$ so that

$$
\text { for each } k \in\left\{0,1, \ldots, 4^{n}-1\right\}, \quad \Delta\left(J_{k}^{n}\right)=\operatorname{diam}\left(E_{k}^{n}\right) .
$$

Fix any $J=J_{k}^{n}$ and consider its four children $J_{0}, J_{1}, J_{2}, J_{3}$. Since $\Delta$ is a 4 -adic diameter function (constructed with the snowflake parameter $p$ ),

$$
\begin{aligned}
\text { either } \quad \Delta\left(J_{0}\right)=\Delta\left(J_{1}\right)=\Delta\left(J_{2}\right)=\Delta\left(J_{3}\right):=\frac{1}{4} \Delta(J) \\
\text { or } \quad \Delta\left(J_{0}\right)=\Delta\left(J_{1}\right)=\Delta\left(J_{2}\right)=\Delta\left(J_{3}\right):=p \Delta(J) .
\end{aligned}
$$

In the first case, we replace the edge $E_{k}^{n}$ with the four segments $E_{4 k}^{n+1}, E_{4 k+1}^{n+1}$, $E_{4 k+2}^{n+1}, E_{4 k+3}^{n+1}$ obtained by dividing $E_{k}^{n}$ into four line segments of equal diameter. Thus here $\operatorname{diam}\left(E_{j}^{n+1}\right)=(1 / 4) \operatorname{diam}\left(E_{k}^{n}\right)$. In the second case, we replace $E_{k}^{n}$ by a similarity copy of the polygonal arc $A_{p}$ pictured at the top right of Figure 1 ; again $E_{k}^{n}$ is replaced by four new segments $E_{j}^{n+1}$, but now each of these has $\operatorname{diameter} \operatorname{diam}\left(E_{j}^{n+1}\right)=p \operatorname{diam}\left(E_{k}^{n}\right)$. The second type of replacement is done so that the "tip" of the replacement arc points into the exterior of $R^{n}$.

It is now straightforward to check that

$$
\text { for each } k \in\left\{0,1, \ldots, 4^{n+1}-1\right\}, \quad \Delta\left(J_{k}^{n+1}\right)=\operatorname{diam}\left(E_{k}^{n+1}\right) .
$$

In particular, we can iterate this construction and thus obtain a sequence $\left(R^{n}\right)_{1}^{\infty}$ of planar polygons. As explained above, the sequence $\left(R^{n}\right)_{1}^{\infty}$ converges, in the Hausdorff metric, to a Rohde snowflake $R$ that has been constructed using the snowflake parameter $p$.

Let $\mathrm{S}^{1} \stackrel{\varphi}{\rightarrow} R$ be the natural homeomorphism induced by the correspondences between the 4 -adic subarcs of $\mathrm{S}^{1}$, all 4 -adic edges, and the 4 -adic subarcs of $R$ (see 
the paragraphs just before (4.5)). Thus each 4-adic edge $E_{k}^{n}$ (of $R^{n}$ ) corresponds to a 4 -adic subarc $A_{k}^{n}=R\left(E_{k}^{n}\right)=\varphi\left(J_{k}^{n}\right)$ of $R$ and

$$
\operatorname{diam}\left(A_{k}^{n}\right)=\operatorname{diam}\left(T\left(E_{k}^{n}\right)\right)=\operatorname{diam}\left(E_{k}^{n}\right)=\Delta\left(J_{k}^{n}\right) .
$$

We claim that $\left(\mathrm{S}^{1}, d_{p}\right) \stackrel{\varphi}{\rightarrow}(R,|\cdot|)$ is bi-Lipschitz with

$$
[c(p) / 8] d_{p}(s, t) \leq|\varphi(s)-\varphi(t)| \leq 8 d_{p}(s, t) \quad \text { for all } s, t \in \mathrm{S}^{1}
$$

where $c(p):=\frac{1}{2}-p$.

To verify this claim, let $s, t$ be two points in $S^{1}$ and write $[s, t]$ for the smaller diameter subarc of $\mathrm{S}^{1}$ joining $s, t$. Appealing to Lemma 3.5, we get 4-adic sub$\operatorname{arcs} I, J$ of $\mathrm{S}^{1}$ such that:

$$
\begin{aligned}
& I \cup J \subset[s, t] \subset \hat{I} \cup \hat{J}, \\
& \Delta(I) \leq \operatorname{diam}_{d_{p}}([s, t])=d_{p}(s, t) \leq 8 \Delta(I), \\
& \Delta(I) \text { is maximal among all } 4 \text {-adic subarcs in }[s, t], \\
& \text { either } I=J \text { or } \hat{I}, \hat{J} \text { are adjacent subarcs. }
\end{aligned}
$$

Here $\hat{I}, \hat{J}$ are the 4 -adic parents of $I, J$. Put $x:=\varphi(s), y:=\varphi(t)$. Let $A:=$ $\varphi(I), B:=\varphi(J)$ and $E, F$ be the 4-adic subarcs of $R$ and 4-adic edges (respectively) that correspond to $I, J$; also, $\hat{A}=\varphi(\hat{I}), \hat{B}=\varphi(\hat{J})$ are the parents of $A, B$.

Since $x, y \in \hat{A} \cup \hat{B}$,

$$
\begin{aligned}
|x-y| & \leq \operatorname{diam}(\hat{A} \cup \hat{B}) \leq \operatorname{diam}(\hat{A})+\operatorname{diam}(\hat{B})=\Delta(\hat{I})+\Delta(\hat{J}) \\
& \leq 4[\Delta(I)+\Delta(J)] \leq 8 \Delta(I) \leq 8 d_{p}(s, t)
\end{aligned}
$$

which establishes the upper estimate in (4.8). To prove the lower estimate in (4.8), we observe that $\operatorname{int}(I)$ separates $s, t$ in $\hat{I} \cup \hat{J}$ and thus (4.7) yields

$$
|x-y| \geq c(p) \operatorname{diam}(\varphi(I))=c(p) \Delta(I) \geq[c(p) / 8] d_{p}(s, t) .
$$

It is worthwhile to observe that the above provides an independent proof that each Rohde snowflake is a quasicircle; in fact, each $R$ in $\mathcal{R}_{p}$ is $C$-bounded turning with $C=C(p):=8 / c(p)=16 /(1-2 p)$.

We close this paper by explaining how Rohde's theorem follows from our theorem. From the proof of part (C) of our theorem, each planar quasicircle is biLipschitz equivalent to a Rohde snowflake. Therefore, Rohde's theorem follows from the fact that a bi-Lipschitz homeomorphism between planar quasicircles has a bi-Lipschitz extension to the entire plane. Below we state this extension theorem, due to Gehring, as Theorem 4.4; see Theorem 7 and Corollary 2 in [8]. The construction of the extension essentially follows from the Beurling-Ahlfors extension [5]. See also Lemma 3 in [18] and Theorems 2.12 and 2.19 in [21].

Interestingly, the property of there being such a bi-Lipschitz extension, for every bi-Lipschitz self-homeomorphism, is a characteristic property of quasicircles among all closed (that is, bounded, so compact) planar Jordan curves. See Theorem 5.1 in $[9]$. 
Theorem 4.4 ([8]). Each bi-Lipschitz homeomorphism between planar quasicircles extends to a bi-Lipschitz self-homeomorphism of the plane. The bi-Lipschitz constant for the extension depends only on the original bi-Lipschitz constant and the two original bounded turning constants.

We end by remarking that the previous theorem is false for Jordan curves. Namely a bi-Lipschitz map between planar Jordan curves $\Gamma_{1}, \Gamma_{2}$ need not have a bi-Lipschitz extension to the plane. For example let $\Gamma_{1}$ be a circle with two outward pointing cusps and let $\Gamma_{2}$ be a circle with one outward and one inward pointing cusp. It is elementary that $\Gamma_{1}$ and $\Gamma_{2}$ are bi-Lipschitz equivalent, but any such map cannot be extended to a bi-Lipschitz map of the whole plane. This example appears already on page 388 in [15].

Acknowledgements. Saara Lehto and David Freeman helped the authors to understand Steffen Rohde's paper. Jussi Väisälä provided many helpful suggestions and references.

\section{References}

[1] Ahlfors, L. V.: Quasiconformal reflections. Acta Math. 109 (1963), 291-301.

[2] Alt, F. And Beer, G.: Der $n$-Gittersatz in Bogen. Ergebnisse math. Koll. 6 (1935), 7.

[3] Assound, P.: Espaces métriques, plongements, facteurs. Thèse de doctorat. Publications Mathématiques d'Orsay, No. 223-7769. U.E.R. Mathématique, Université Paris XI, Orsay, 1977.

[4] Assound, P.: Étude d'une dimension métrique liée à la possibilité de plongements dans $\mathbb{R}^{n}$. C. R. Acad. Sci. Paris Sér. A-B 288 (1979), no. 15, A731-A734.

[5] Beurling, A. And Ahlfors, L. V.: The boundary correspondence under quasiconformal mappings. Acta Math. 96 (1956), 125-142.

[6] Bonk, M., Heinonen, J. And Rohde, S.: Doubling conformal densities. J. Reine Angew. Math. 541 (2001), 117-141.

[7] Gehring, F. W.: Characteristic properties of quasidisks. Séminaire de Mathématiques Supérieures, 84. Les Presses de l'Université de Montréal, Montréal, Quebec, 1982.

[8] Gehring, F. W.: Injectivity of local quasi-isometries. Comment. Math. Helv. 57 (1982), no. 2, 202-220.

[9] Gehring, F. W.: Extension of quasi-isometric embeddings of Jordan curves. Complex Variables Theory Appl. 5 (1986), no. 2-4, 245-263.

[10] Heinonen, J.: Lectures on analysis on metric spaces. Universitext. Springer-Verlag, New York, 2001.

[11] Luukkainen, J.: Assouad dimension: antifractal metrization, porous sets, and homogeneous measures. J. Korean Math. Soc. 35 (1998), no. 1, 23-76.

[12] Menger, K.: Untersuchungen über allgemeine Metrik. Math. Ann. 103 (1930), no. $1,466-501$.

[13] Meyer, D.: Bounded turning circles are weak-quasicircles. Proc. Amer. Math. Soc. 139 (2011), no. 5, 1751-1761. 
[14] Näkki, R. ANd VÄıs̈̈LÄ, J.: John disks. Exposition. Math. 9 (1991), no. 1, 3-43.

[15] Rickman, S.: Quasiconformally equivalent curves. Duke Math. J. 36 (1969), 387-400.

[16] Rohde, S.: Quasicircles modulo bilipschitz maps. Rev. Mat. Iberoamericana 17 (2001), no. 3, 643-659.

[17] Schoenberg, I. J.: On metric arcs of vanishing Menger curvature. Ann. of Math. (2) 41 (1940), 715-726.

[18] Tukia, P.: Extension of quasisymmetric and Lipschitz embeddings of the real line into the plane. Ann. Acad. Sci. Fenn. Ser. A I Math. 6 (1981), no. 1, 89-94.

[19] Tukia, P.: Spaces and arcs of bounded turning. Michigan Math. J. 43 (1996), no. 3, $559-584$.

[20] Tukia, P. And VÄIs⿱̈亡̈̈, J.: Quasisymmetric embeddings of metric spaces. Ann. Acad. Sci. Fenn. Ser. A I Math. 5 (1980), no. 1, 97-114.

[21] Tukia, P. ANd VÄIsÄLÄ, J.: Bi-Lipschitz extensions of maps having quasiconformal extensions. Math. Ann. 269 (1984), no. 4, 561-572.

[22] VÄıs̈̈LÄ, J.: Dividing an arc to subarcs with equal chords. Colloq. Math. 46 (1982), no. 2, 203-204.

Received July 1, 2010.

DAvid A. Herron: Department of Mathematics, University of Cincinnati, OH 45221, USA.

E-mail: David.Herron@UC.edu

Daniel Meyer: Department of Mathematics and Statistics, University of Helsinki, P.O. Box 68 (Gustaf Hällströmin katu 2b), 00014, Helsinki, Finland.

E-mail: DMeyermail@gmail.com

The first author was partially supported by the Charles Phelps Taft Research Center. The second author was supported by the Academy of Finland, projects SA-134757 and SA-118634. 